\title{
Calcium/calmodulin-dependent protein kinase II links ER stress with Fas and mitochondrial apoptosis pathways
}

\author{
Jenelle M. Timmins, ${ }^{1}$ Lale Ozcan, ${ }^{1}$ Tracie A. Seimon, ${ }^{1}$ Gang $\mathrm{Li},{ }^{1}$ Cristina Malagelada, ${ }^{2,3}$
} Johannes Backs, ${ }^{4}$ Thea Backs, ${ }^{5}$ Rhonda Bassel-Duby, ${ }^{5}$ Eric N. Olson, ${ }^{5}$ Mark E. Anderson, ${ }^{6}$ and Ira Tabas ${ }^{1,2,7}$

\begin{abstract}
'Department of Medicine, ${ }^{2}$ Department of Pathology and Cell Biology, and ${ }^{3}$ Center for Neurobiology and Behavior and Taub Center for Alzheimer's Disease Research, Columbia University, New York, New York, USA. ${ }^{4}$ Department of Internal Medicine III, University of Heidelberg, Heidelberg, Germany. ${ }^{5}$ Department of Molecular Biology, University of Texas Southwestern Medical Center, Dallas, Texas, USA.

${ }^{6}$ Department of Internal Medicine, Division of Cardiovascular Medicine, and Department of Physiology and Molecular Biophysics, University of lowa, Carver College of Medicine, lowa City, lowa, USA. 'Department of Physiology and Cellular Biophysics, Columbia University, New York, New York, USA.
\end{abstract}

\begin{abstract}
ER stress-induced apoptosis is implicated in various pathological conditions, but the mechanisms linking ER stress-mediated signaling to downstream apoptotic pathways remain unclear. Using human and mouse cell culture and in vivo mouse models of ER stress-induced apoptosis, we have shown that cytosolic calcium resulting from ER stress induces expression of the Fas death receptor through a pathway involving calcium/ calmodulin-dependent protein kinase II $\gamma($ CaMKII $\gamma)$ and JNK. Remarkably, CaMKII $\gamma$ was also responsible for processes involved in mitochondrial-dependent apoptosis, including release of mitochondrial cytochrome c and loss of mitochondrial membrane potential. CaMKII-dependent apoptosis was also observed in a number of cultured human and mouse cells relevant to ER stress-induced pathology, including cultured macrophages, endothelial cells, and neuronal cells subjected to proapoptotic ER stress. Moreover, WT mice subjected to systemic ER stress showed evidence of macrophage mitochondrial dysfunction and apoptosis, renal epithelial cell apoptosis, and renal dysfunction, and these effects were markedly reduced in CaMKII $\gamma$-deficient mice. These data support an integrated model in which CaMKII serves as a unifying link between ER stress and the Fas and mitochondrial apoptotic pathways. Our study also revealed what we believe to be a novel proapoptotic function for CaMKII, namely, promotion of mitochondrial calcium uptake. These findings raise the possibility that CaMKII inhibitors could be useful in preventing apoptosis in pathological settings involving ER stress-induced apoptosis.
\end{abstract}

\section{Introduction}

The unfolded protein response (UPR) is an ER stress-induced signal transduction pathway that responds to perturbations of ER homeostasis with a number of corrective functions $(1,2)$. When ER stress is prolonged, however, apoptosis can occur $(3,4)$, and this process has been implicated in a wide variety of pathological conditions (5-8). Many of the mechanistic studies in this area have focused on the proapoptotic role of the UPR effector C/EBP homologous protein (CHOP; also known as GADD153; refs. 3, 4). However, there are many areas of uncertainty related to mechanisms of ER stress-induced apoptosis, particularly in scenarios that are relevant to actual disease processes.

A prime example in which ER stress-induced cell death plays an important role in disease is macrophage apoptosis in advanced atherosclerotic lesions. Advanced lesional macrophage death leads to plaque necrosis, a key determinant of plaque disruption and acute cardiovascular disease (9). In vitro, macrophages exposed to a number of atherosclerosis-relevant ER stressors, such as excess lipoprotein-derived cholesterol or oxysterols, undergo apoptosis triggered by both death receptor and mitochondrial dysfunction pathways

Authorship note: Jenelle M. Timmins and Lale Ozcan contributed equally to this work.

Conflict of interest: The authors have declared that no conflict of interest exists. Citation for this article: J. Clin. Invest. 119:2925-2941 (2009). doi:10.1172/JCI38857.
(3, 10-12). In vivo, studies using models of advanced lesional macrophage death have shown that the UPR is activated and necessary for cell death $(3,13-15)$. Moreover, UPR activators are known to be present in advanced atherosclerotic lesions (9), and macrophages in advanced murine and human lesions show signs of UPR activation that correlate very closely with lesional macrophage apoptosis and plaque disruption $(3,16-18)$. Most importantly, prevention of the UPR by genetic engineering in mice decreases advanced lesional macrophage apoptosis and plaque necrosis, and vice versa $(13,19,20)$.

A major gap in our knowledge of UPR-induced apoptosis is how ER stress-mediated events are linked to the actual executers of programmed cell death. Knowledge gained in this area will likely apply to the broader issue of UPR-induced apoptosis and the diseases associated with this event $(6-8,21)$. For example, certain forms of ER stress-induced apoptosis, including that induced by $\beta$-amyloid in neurons, involves activation of caspase-12 (22). In cases of ER stressinduced macrophage death in vivo, apoptosis likely occurs through the combination of relatively subtle, subapoptotic UPR activation with 1 or more additional proapoptotic hits. For example, in vitro and in vivo studies have provided evidence that pattern recognition receptor (PRR) ligands may synergize with ER stressors to induce macrophage apoptosis in advanced atherosclerotic plaques $(12,15$, $23,24)$. UPR activation in these cells triggers a number of processes necessary for apoptosis, notably release of ER calcium stores into the cytosol, activation of the calcium-signal transducer CaMKII, 
A

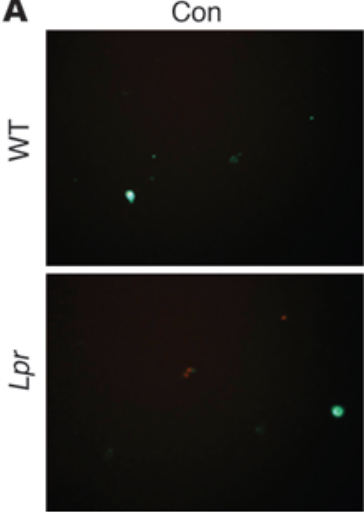

B

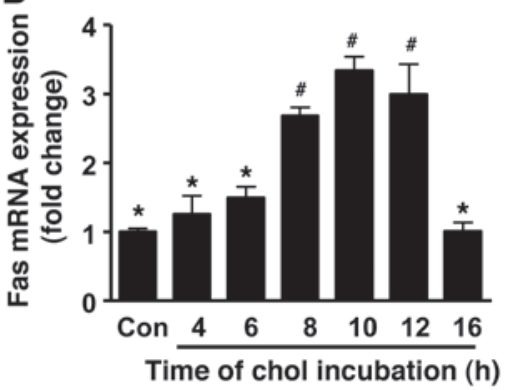

C
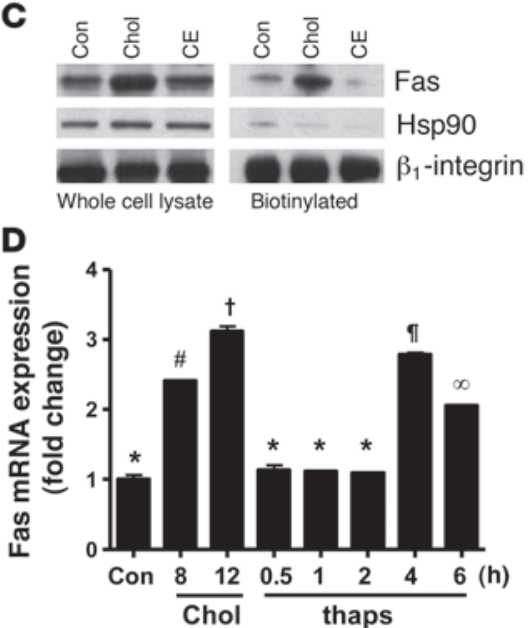

E

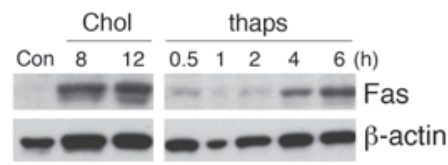

Chol
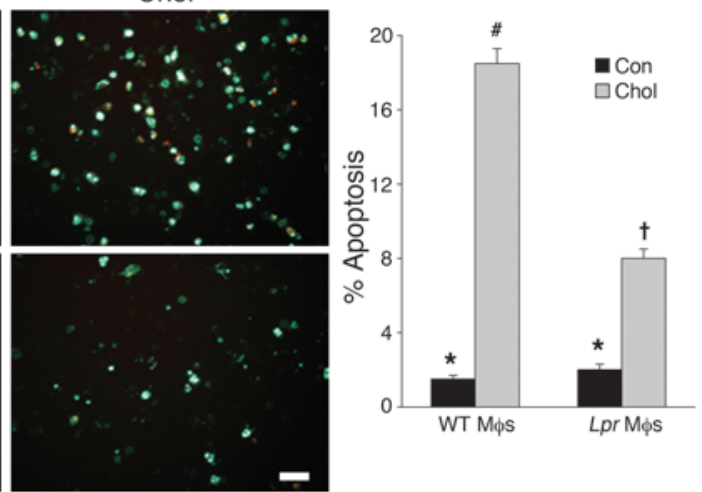

$\mathbf{F}$

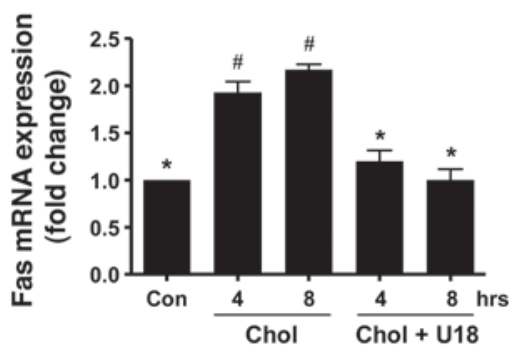

G

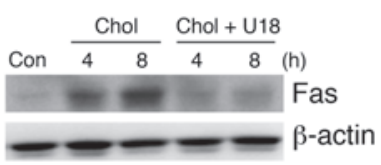

H

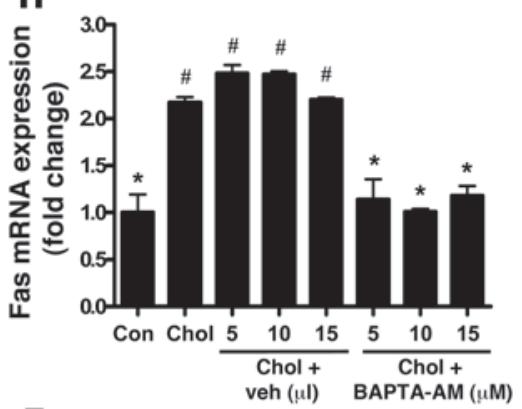

$\mathbf{F}$

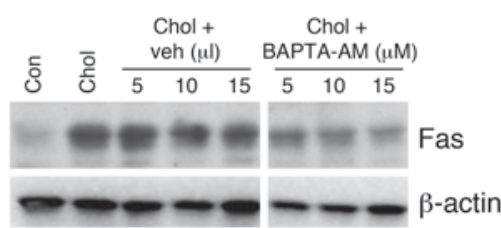

Figure 1

Proapoptotic Fas is induced by ER stress in a calcium-dependent manner. (A) Peritoneal macrophages from WT or Lpr mice were incubated for 14 hours under control (Con) or cholesterol-loading (Chol) conditions. Mid- and late-stage apoptosis were assayed using Alexa Fluor 488-conjugated annexin V (green) and PI (orange-red), respectively. Scale bar: $20 \mu \mathrm{m}$. For each group, 3 fields were quantified and expressed as a percentage of annexin/PI-positive cells. $\mathrm{M} \phi$, macrophage. (B) Macrophages from WT mice were incubated under cholesterol-loading conditions for the indicated times. Fas mRNA, measured using quantitative PCR, is expressed relative to internal control mRNA. (C) Macrophages were incubated for 8 hours under control, cholesterol-loading, or cholesteryl ester-loading (CE) conditions and then assayed for total and cell surface Fas protein by immunoblot following biotinylation of cell surface proteins. Hsp90 was used as a cytosolic marker, and $\beta_{1}$ integrin as a cell surface marker and loading control. ( $\mathbf{D}$ and $\mathbf{E}$ ) Macrophages were incubated under control or cholesterol-loading conditions or with 0.25 $\mu \mathrm{M}$ thapsigargin (Thaps) and then assayed for Fas mRNA and protein. (F and G) Macrophages were incubated under control or cholesterol-loading conditions in the absence or presence of the cholesterol trafficking inhibitor U18666A (U18) after 1 hour pretreatment with the compound, and then assayed for Fas mRNA and protein. (H and I) Macrophages were incubated under control or cholesterolloading conditions with BAPTA-AM or equivalent volumes of vehicle (Veh) control after 1 hour pretreatment, and then assayed for Fas mRNA and protein. Differing symbols indicate $P<0.01$; identical symbols indicate differences that are not significant. and activation of the mitogen-activated protein kinase JNK, but not activation of caspase- $12(3,15,23,25,26)$. As alluded to above, death itself is executed through both engagement of the Fas death receptor pathway and activation of the mitochondrial death pathway (10, 11). In this context, the goal of the current study was to elucidate mechanistic links between proapoptotic ER stress-mediated processes and the Fas and mitochondrial pathways of apoptosis. We showed that both the Fas pathway, through induction of the Fas receptor itself, and the mitochondrial pathway, through accumula- tion of mitochondrial calcium, were both activated through CaMKII signaling in ER-stressed macrophages. These findings provide an integrated, unified mechanism linking the UPR to apoptosis and suggest a feasible, focused target for intervention directed against pathological ER stress-induced apoptosis.

\section{Results}

Fas, an important protein in macrophage apoptosis during cholesterol loading, is induced by ER stress in a calcium-dependent manner. Our initial 
A

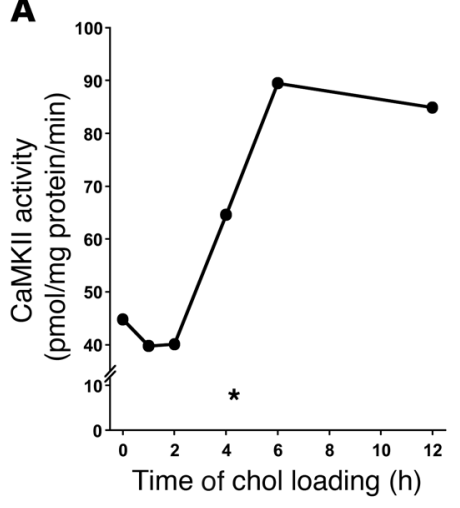

B

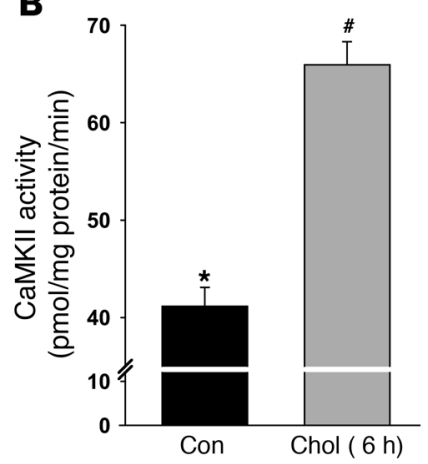

C

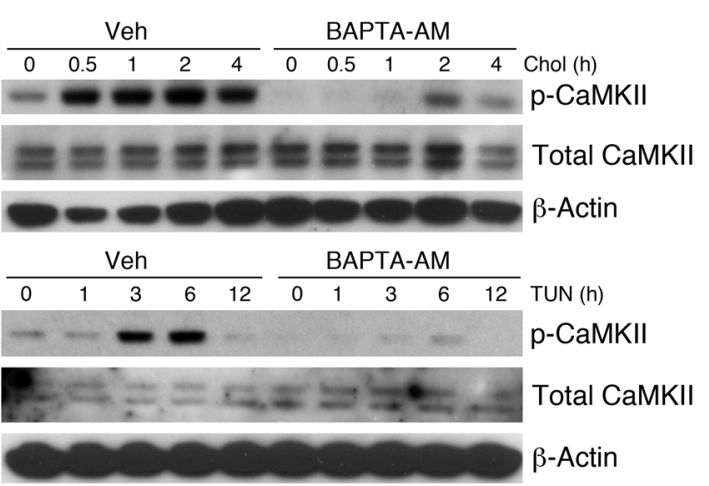

D

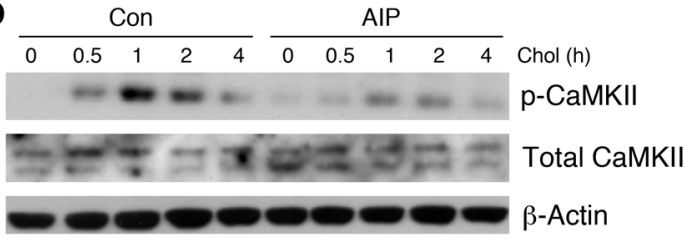

$\mathbf{F}$

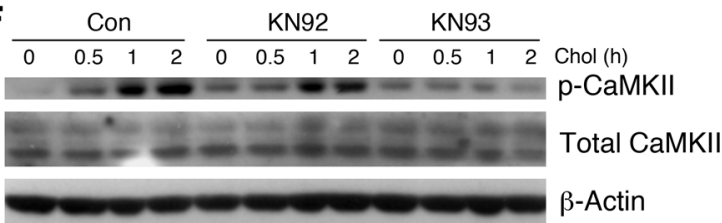

E

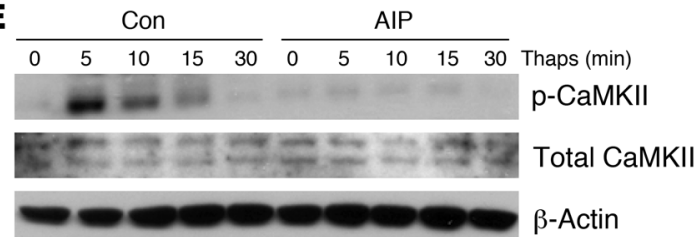

G

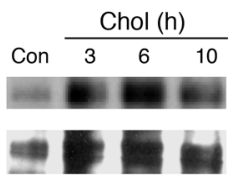

IP: Anti-CaMKII

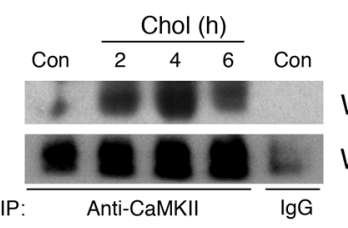

WB: Anti-Ox-CaMKII

WB: Anti-CaMKII

\section{Figure 2}

Cholesterol loading leads to sustained activation of CaMKII. (A) CaMKII activity was assayed in individual wells of macrophages incubated under cholesterol-loading conditions for the indicated times. The asterisk indicates CaMKII activity from cells loaded with cholesterol for 4 hours in the presence of $10 \mu \mathrm{M}$ AIP-II (EGTA was included during the assay). (B) CaMKII activity was assayed in triplicate wells of macrophages incubated under control or cholesterol-loading conditions for 6 hours. Differing symbols indicate $P=0.001$. (C) Top: Macrophages were incubated under control or cholesterol-loading conditions with BAPTA-AM or vehicle after 1 hour pretreatment, and lysates were then immunoblotted for phosphoCaMKII, total CaMKII, and $\beta$-actin. Bottom: As above, except ER stress was induced with $3 \mu \mathrm{g} / \mathrm{ml}$ tunicamycin (Tun). (D) Macrophages were incubated under control or cholesterol-loading conditions with or without $10 \mu \mathrm{M}$ AIP-II after 1 hour pretreatment, and lysates were then immunoblotted for phospho-CaMKII, total CaMKII, and $\beta$-actin. (E and F) As in $\mathbf{D}$, except that (E) ER stress was induced with $0.25 \mu \mathrm{M}$ thapsigargin, or $(\mathbf{F}) 10$ uM KN93 was used as the CaMKII inhibitor, with $10 \mu \mathrm{M}$ KN92 as the inactive analog. (G) In 2 independent experiments, macrophages were incubated under control or cholesterol-loading conditions. CaMKII was then immunoprecipitated from cell lysates using anti-CaMKII or control IgG (bottom). The immunoprecipitates were then blotted for oxidized CaMKII using anti-Ox-CaMKII antiserum and total CaMKII.

goal was to determine whether there is a mechanistic link between 2 processes previously shown to be involved in ER stress-induced macrophage apoptosis, namely, calcium-CaMKII signaling and activation of the Fas pathway $(10,23,25)$. As a model of ER stressinduced apoptosis, macrophages were incubated under conditions promoting the accumulation of lipoprotein-derived cholesterol (3, $10,11)$. In our original Fas study (10), a serum-deprivation component was included in the model, but we have since cultured the macrophages in full serum in order to simplify the conditions. In the current model, we first determined the role of Fas in apoptosis. Macrophages from Lpr mice, which lack Fas receptors, showed a $60 \%$ reduction in cholesterol-induced apoptosis compared with macrophages from WT mice $(P=0.01$; Figure $1 \mathrm{~A})$. Inhibition of caspase-8, the downstream caspase of Fas, with Z-Ile-Glu(OMe)Thr-Asp-fluoromethylketone inhibited cholesterol-induced apoptosis from $17.9 \% \pm 1.5 \%$ to $8.5 \% \pm 0.9 \%(P<0.001)$. We then tested whether cholesterol loading affected expression of Fas itself, and found that cholesterol loading led to a marked induction of Fas mRNA (Figure 1B) and total and cell surface Fas protein (Figure
1C). The latter finding, which was obtained using a cell surface biotinylation protocol, is important because Fas is active in apoptotic signaling as a cell-surface receptor (27). Thus, cholesterol loading of macrophages induces Fas expression, which plays a proapoptotic role in these cells.

Lipoprotein-mediated cholesterol loading induces apoptosis through 2 proapoptotic hits: ER stress induced by lipoproteinderived cholesterol and additional death signaling through engagement of PRRs by the lipoproteins themselves $(12,15$, 23). The first evidence that the ER stress hit was important in Fas induction was our finding in Figure 1C that induction of Fas protein was not observed in macrophages incubated under conditions in which lipoprotein cholesterol is efficiently esterified to fatty acids, which do not perturb the ER membrane and thus do not cause ER stress (3). Second, we found that the sarcoplasmic/endoplasmic reticulum calcium-dependent ATPase (SERCA) inhibitor thapsigargin, a UPR activator, induced Fas mRNA and protein (Figure 1, D and E). Third, we took advantage of the fact that the steroid $3 \beta$-[2-(diethylamino)ethoxy]- 
A

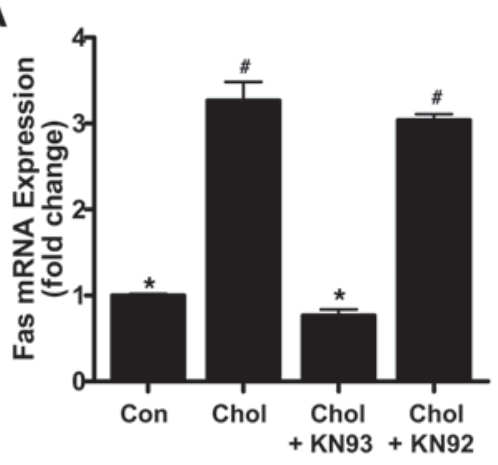

B
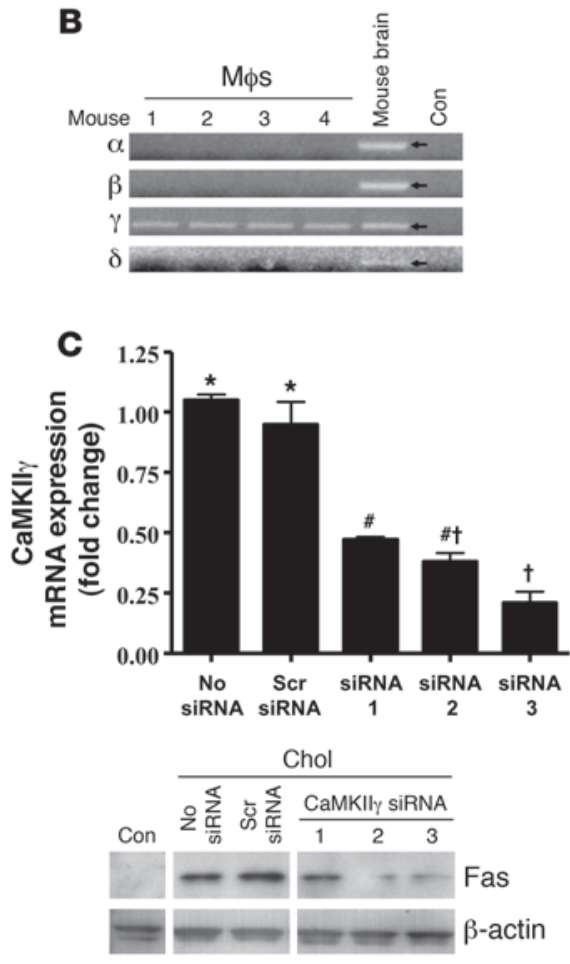

D

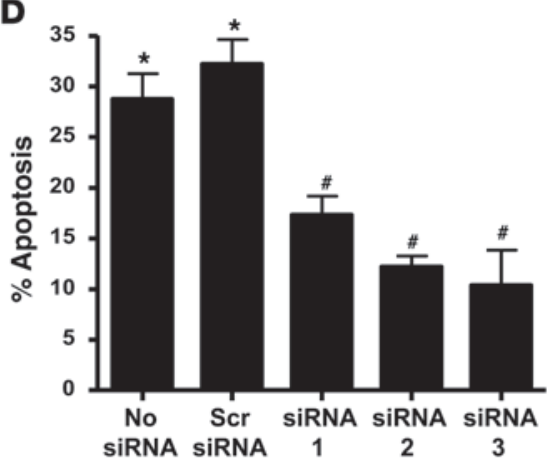

E
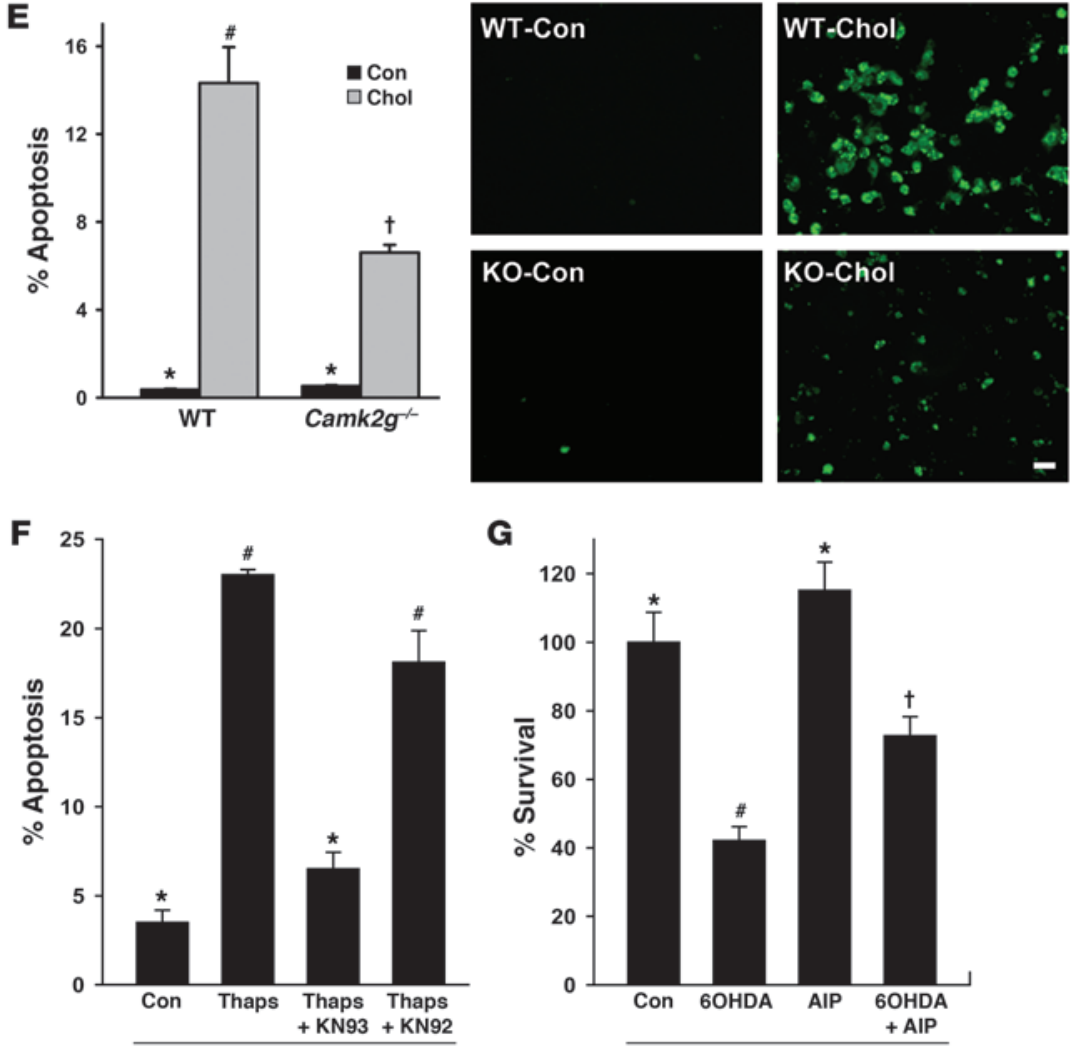

Endothelial cells
G

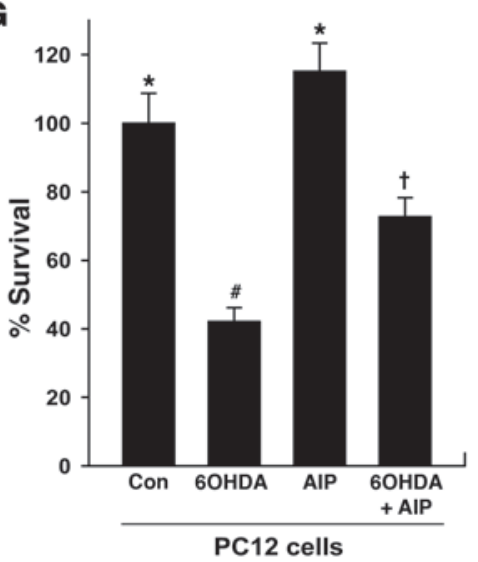

\section{Figure 3}

Induction of Fas and apoptosis by ER stress involves CaMKII. (A) Macrophages were incubated for 8 hours under cholesterol-loading conditions with or without KN93 or KN92 after 1 hour pretreatment, and then assayed for Fas mRNA. (B) RNA from peritoneal macrophages from 4 separate mice and from mouse brain, along with water control, were probed for the indicated CaMKII isoform mRNAs by RT-PCR. (C) Macrophages were transfected with 3 different CaMKIl $\gamma$ siRNA constructs. After 72 hours, the cells were incubated for 8 hours under cholesterol-loading conditions and then assayed for CaMKIl $\gamma$ mRNA and total Fas protein. (D) Macrophages were transfected with the 3 siRNA constructs in C, incubated for 30 hours with $0.25 \mu \mathrm{M}$ thapsigargin and $25 \mu \mathrm{g} / \mathrm{ml}$ of the SRA ligand fucoidan, and then assayed for apoptosis. (E) Macrophages from WT or Camk $2 \mathrm{~g}^{-/-}$mice were incubated under control or cholesterol-loading conditions for 12 hours and then assayed for apoptosis. Scale bar: 20 um. (F) Human aortic endothelial cells were incubated for 24 hours with thapsigargin (1 $\mu \mathrm{M})$ with or without KN93 or KN92 after 1 hour pretreatment, and then assayed for apoptosis. (G) PC12 cells were incubated for 24 hours with $100 \mu \mathrm{M}$ 6-OHDA with or without $10 \mu \mathrm{M}$ AIP-II after 1 hour pretreatment, and then assayed for cell viability (percentage of viable cells compared with those in cultures not treated with 6-OHDA). Differing symbols indicate $P<0.01$; identical symbols indicate differences that are not significant.

androst-5-en-17-one hydrochloride (U18666A) blocks the trafficking of lipoprotein-derived cholesterol from late endosomes to the ER and thus prevents cholesterol-induced ER stress and apoptosis (3). Loading macrophages with cholesterol in the presence of U18666A suppressed Fas mRNA and protein to basal levels (Figure 1, F and G). As a control, we showed that Fas induction by LPS, a non-ER stress inducer of Fas (28), was not blocked by U18666A (data not shown). Thus, ER stress is essential for Fas induction during enrichment of macrophages with lipoprotein-cholesterol. 


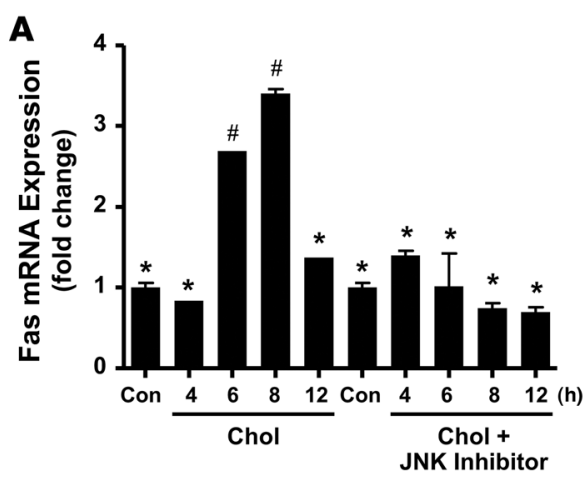

B

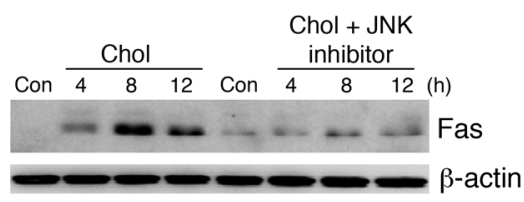

C

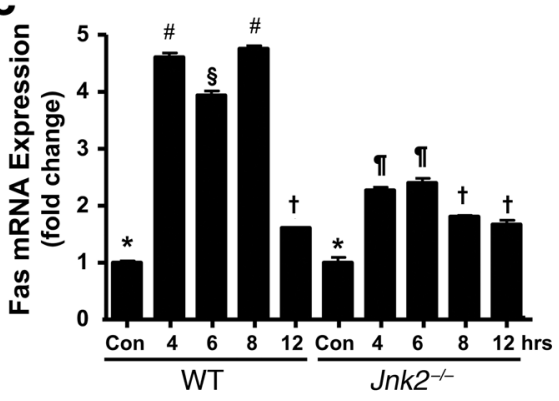

D

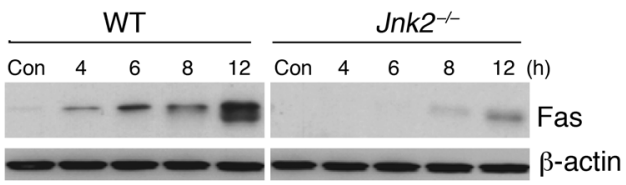

E

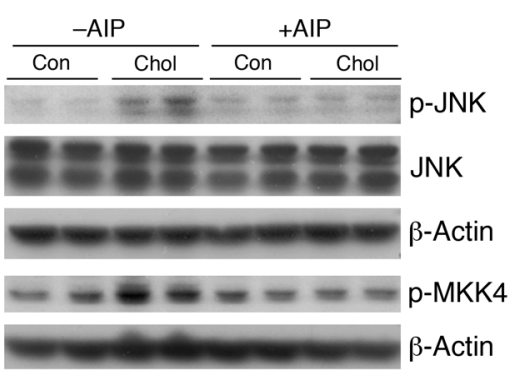

$\mathbf{F}$
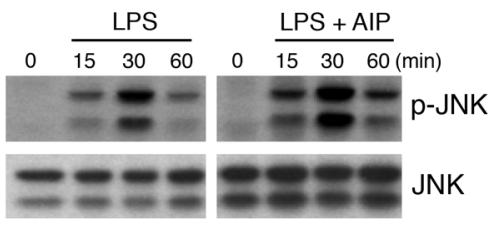

G

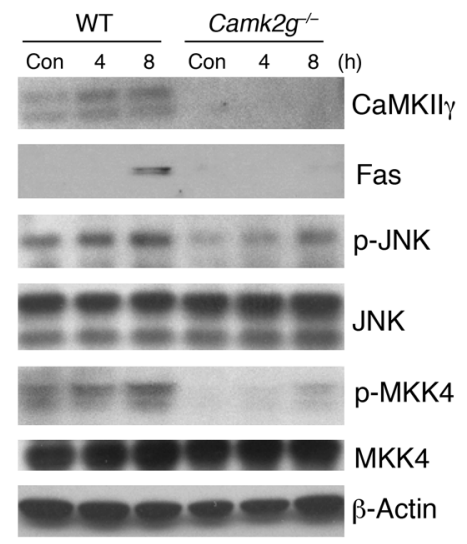

Figure 4

JNK mediates the proapoptotic role of CaMKII in ER-stressed macrophages. (A and $\mathbf{B}$ ) Macrophages were incubated under control or cholesterol-loading conditions with or without $10 \mu \mathrm{M}$ of the JNK inhibitor SP600125 after 1 hour pretreatment, and then assayed for Fas mRNA (A) and protein (B). (C and D) Macrophages from WT or Jnk2-/- mice were incubated under control or cholesterol-loading conditions and then assayed for Fas mRNA (C) and protein (D). (E) Macrophages were incubated under control or cholesterol-loading conditions for 4 hours with or without $10 \mu \mathrm{M}$ AIP-II after 1 hour pretreatment. One aliquot of the cell extracts was immunoblotted for phospho-JNK, total JNK, and $\beta$-actin, and another was immunoblotted for phospho-MKK4 and $\beta$-actin. (F) Macrophages were incubated with $100 \mathrm{ng} / \mathrm{ml}$ LPS for the indicated times with or without $10 \mu \mathrm{M}$ AIP-II after 1 hour pretreatment. Phospho-JNK and total JNK were then assayed by immunoblot. (G) Macrophages from WT or Camk2 $\mathrm{g}^{-1-}$ mice were incubated under control or cholesterol-loading conditions and then immunoblotted for CaMKII, Fas, phosphoJNK, JNK, phospho-MKK4, MKK4, and $\beta$-actin. Differing symbols indicate $P<0.01$; identical symbols indicate differences that are not significant.
A critical mechanism by which ER stress enables apoptosis in macrophages is through release of ER calcium stores into the cytosol $(23,25)$. To determine the role of cytosolic calcium in cholesterol-mediated Fas induction, macrophages were loaded with cholesterol in the absence or presence of a cytosolic calcium chelator, 1,2-bis(2-aminophenoxy)ethane- $N, N, N^{\prime}, N^{\prime}$-tetraacetate-AM (BAPTA-AM). Our previous work showed that BAPTA-AM blocks cholesterol-induced apoptosis (23). BAPTA-AM almost completely prevented Fas mRNA and protein induction (Figure 1, H and I), suggesting a critical role for cytosolic calcium in this process. In summary, ER stress, including that induced by cholesterol loading, induces Fas, which is an important protein in ER stress-induced macrophage apoptosis. Cytosolic calcium, a key agent in ER stressinduced apoptosis $(23,25)$, is required for this process.

Cholesterol loading leads to sustained activation of CaMKII. CaMKII is a critical signal transducer in ER stress-induced macrophage apoptosis (25) and thus was a candidate for mediating calciumdependent induction of Fas. We first sought to firmly establish that CaMKII was activated in this model. Macrophages were incubated under cholesterol-loading conditions for various times, and then cell extracts were assayed for their ability to transfer ${ }^{32} \mathrm{P}$ from $\left[\gamma-{ }^{32} \mathrm{P}\right]$ ATP to the CaMKII-specific substrate, KKALRRQETVDAL (29). CaMKII activity increased steadily as a function of time of cholesterol loading up to 7 hours (Figure 2A). As a negative con- trol, 4-hour cells were incubated with the specific CaMKII peptide inhibitor autocamtide-2-related inhibitory peptide II (AIP-II), which contains the sequence of the Thr287-containing autophosphorylation domain and thus acts as a competitive inhibitor of autophosphorylation (30); and the calcium chelator EGTA was added in vitro to the enzyme reaction mix. CaMKII activity was very low under these conditions (Figure 2A, asterisk). A repeat experiment at the 6-hour time point again showed activation of the enzyme (Figure 2B).

CaMKII is initially activated by calcium/calmodulin through transient increases in cytosolic calcium, leading to sustained, calcium-independent activity due to inactivation of the autoinhibitory domain of the enzyme through autophosphorylation at Thr287 (31). Thus, another measure of CaMKII activation is the appearance of phospho-Thr287 CaMKII. There was an increase in phospho-Thr287 CaMKII at relatively early time points of cholesterol loading (Figure 2C), consistent with this process leading to sustained enzyme activation at later time points (see above and ref. 25). Although there was some variability among experiments as to the exact timing of onset and duration of phospho-CaMKII after cholesterol loading, we consistently observed its appearance within 30-60 minutes of cholesterol loading, with a duration of 2-6 hours. Another inducer of ER stress is the glycosylation inhibitor tunicamycin, and treatment of macrophages with this reagent 


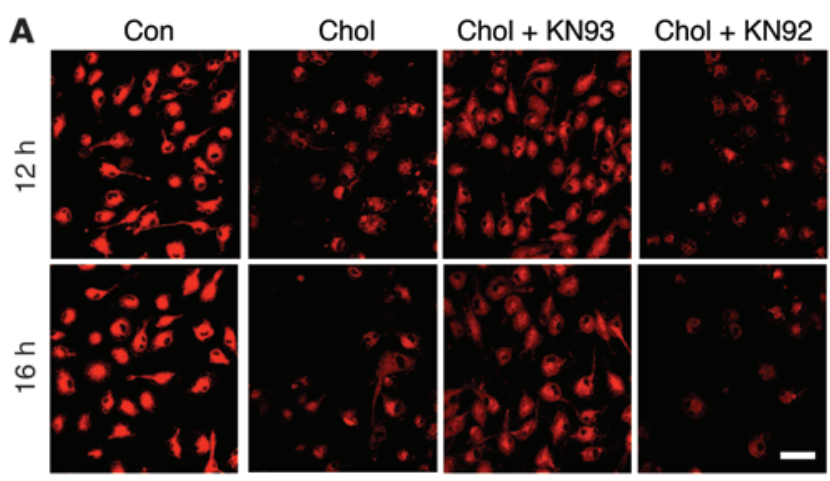

MitoTracker Red
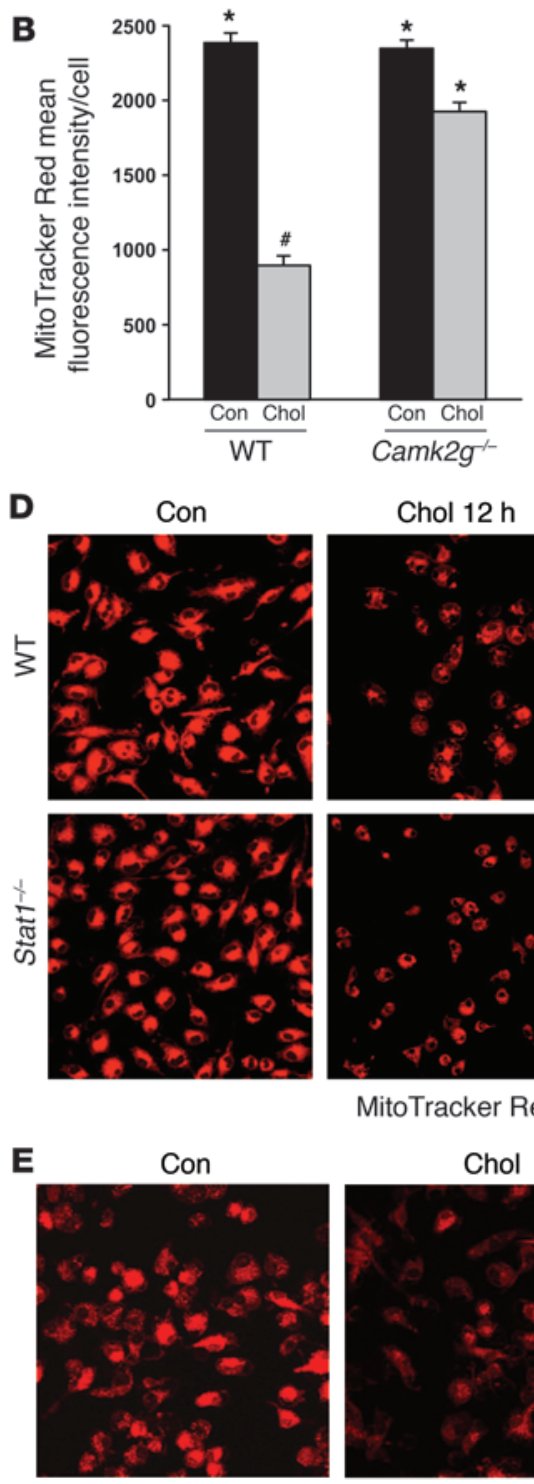

C

Chol $16 \mathrm{~h}$

MitoTracker Red

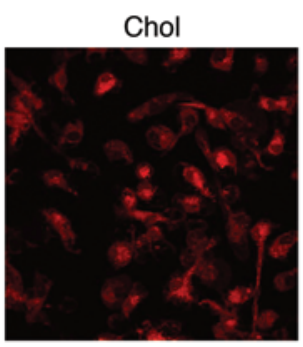

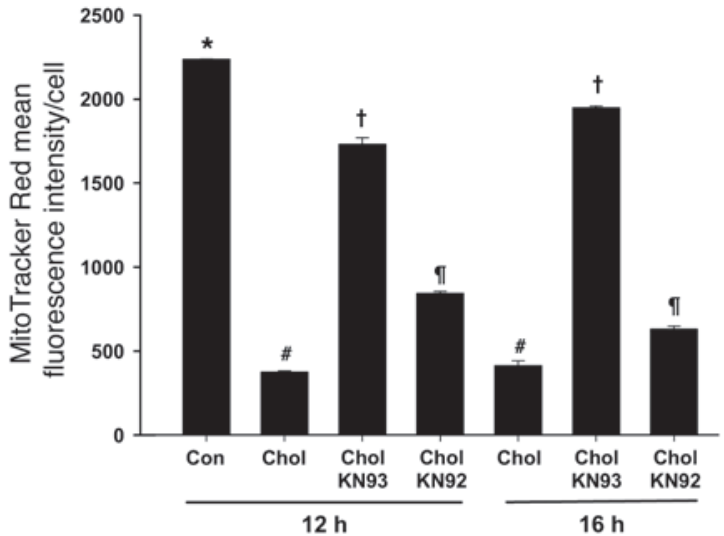
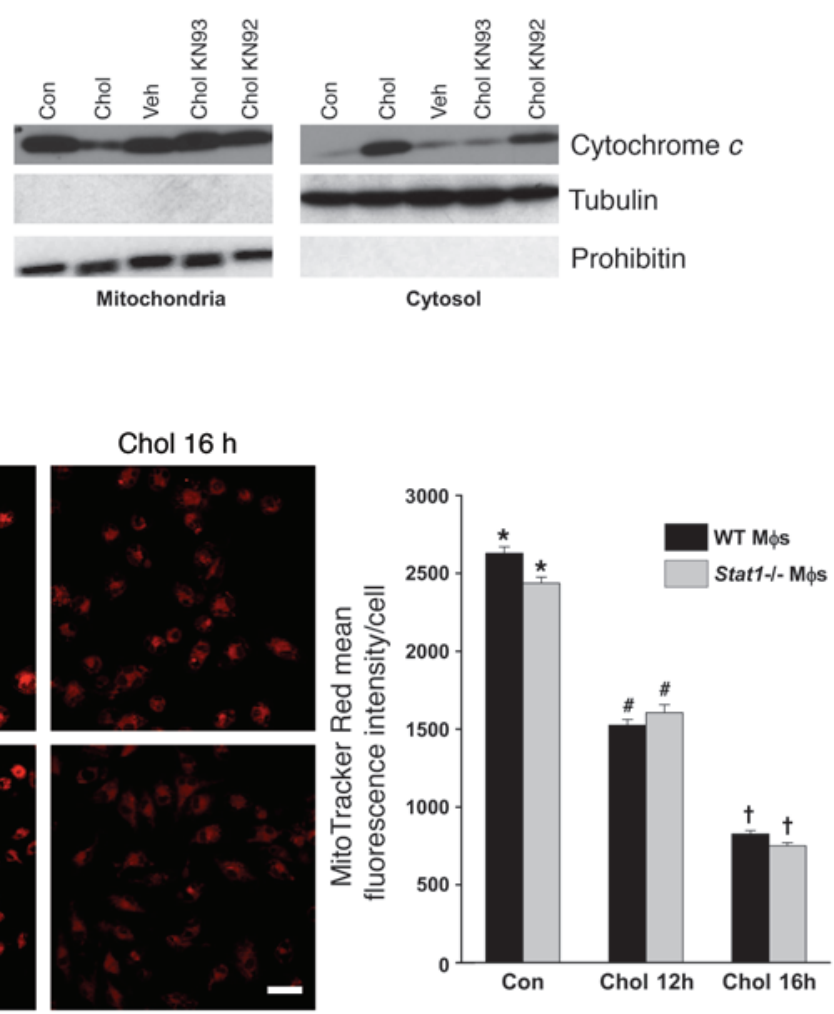

MitoTracker Red
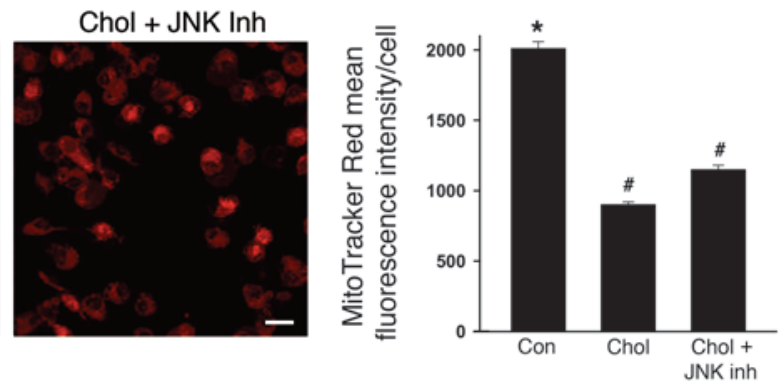


\section{Figure 5}

Release of mitochondrial cytochrome $\mathrm{c}$ and $\Delta \psi_{\mathrm{m}}$ in cholesterol-loaded macrophages is dependent on CaMKII. (A) Macrophages were incubated under control or cholesterol-loading conditions for 12 or 16 hours in the absence or presence of $10 \mu \mathrm{M}$ KN93 or KN92 after 1 hour pretreatment. Cells were then stained with MitoTracker Red and examined by confocal fluorescence microscopy. Mean fluorescence intensity per cell is also shown ( $n=100$ cells). (B) Macrophages from WT or Camk $2 \mathrm{~g}^{-1-}$ mice were incubated under control or cholesterol-loading conditions for 12 hours and then assayed for MitoTracker Red staining. (C) Macrophages were treated for 8 hours as in A. Mitochondrial and cytosolic fractions were assayed for cytochrome c, tubulin (cytosolic marker), and prohibitin (mitochondrial marker). (D) Macrophages from WT or Stat $1^{-1-}$ mice were incubated under control or cholesterol-loading conditions for 12 or 16 hours and then stained with MitoTracker Red. (E) Macrophages were incubated under control or cholesterolloading conditions for 16 hours in the absence or presence of $10 \mu \mathrm{M}$ of the JNK inhibitor SP600125 after 1 hour pretreatment, and then stained with MitoTracker Red. Scale bars: $20 \mu \mathrm{m}$ (A); $10 \mu \mathrm{m}$ (D and E). Differing symbols indicate $P<0.01$; identical symbols indicate differences that are not significant.

also caused a marked increased in CaMKII phosphorylation. To validate phospho-CaMKII as a marker of CaMKII activation in our models, we tested the effect of BAPTA-AM, which should block the interaction of calcium with calmodulin and thus prevent phosphorylation of the enzyme. Consistent with this point, we found that BAPTA-AM markedly decreased both cholesterol- and tunicamycin-induced CaMKII phosphorylation (Figure 2C).

We next used phospho-CaMKII to further validate the use of AIP-II as a CaMKII inhibitor (above) as well as another, structurally unrelated CaMKII inhibitor, 2-[N-(2-hydroxyethyl)]-N-(4methoxybenzenesulfonyl)] amino-N-(4-chlorocinnamyl)-Nmethylbenzylamine (KN93), that we used in the following experiments. As expected from the data in Figure 2A, AIP-II blocked both cholesterol- and thapsigargin-induced CaMKII phosphorylation (Figure 2, D and E). Note that CaMKII phosphorylation occurred at a much earlier time point with thapsigargin than with lipoprotein-cholesterol loading, as expected of a direct SERCA inhibitor. Cholesterol-induced CaMKII phosphorylation was also blocked by KN93, but not by its inactive homolog, [N-(4-methoxybenzenesulfonyl)] amino-N-(4-chlorocinnamyl)$\mathrm{N}$-methylbenzylamine phosphate (KN92; Figure 2F). These combined data show that a variety of ER stressors activate CaMKII in macrophages and validate the use of AIP-II and KN93 as CaMKII inhibitors in these models (see below).

Prolonged ER stress can generate intracellular ROS (4), and recent work in our laboratory has shown that cholesterol loading is a potent inducer of ROS in macrophages (G. Li, unpublished observations; T.A. Seimon, unpublished observations). Erickson et al. (32) recently showed that ROS-mediated oxidation of Met281/282 in the autoinhibitory domain of CaMKII further prolongs CaMKII activity after an initial calcium transient by a mechanism analogous to autophosphorylation. We therefore probed immunoprecipitated CaMKII in cholesterol-loaded macrophages with an antibody specific for the oxidized CaMKII regulatory domain peptide M281/282 (Ox-CaMKII). In 3 independent experiments, we found a positive Ox-CaMKII signal after 2 hours of cholesterol loading (Figure 2G). Thus, cholesterol loading leads to sustained activation of CaMKII, which likely involves initial activation by cytosolic calcium followed by prolonged activation through autophosphorylation- and oxidation-mediated modification of the autoinhibitory domain.

Induction of Fas and apoptosis by ER stress involves CaMKII. Given the role of cytosolic calcium in cholesterol-induced Fas induction and the ability of cholesterol loading to activate CaMKII, we addressed the causative role of CaMKII in this process. Inclusion of the CaMKII inhibitor KN93 during cholesterol loading prevented Fas induction, whereas KN92 had no inhibitory effect (Figure $3 \mathrm{~A})$. To further substantiate this important point, we used a CaMKII siRNA knockdown approach. We first showed by RT-PCR that mouse peritoneal macrophages express only the $\gamma$ isoform of CaMKII (Figure 3B) and then found 3 CaMKII $\gamma$ siRNA species that decreased CaMKII expression by 55\%-75\% (Figure 3C). Consistent with the KN93 data, cholesterol-induced Fas protein was suppressed by these siRNA species, whereas a scrambled siRNA had no effect (Figure 3C). Thus, CaMKII activity and expression are necessary for ER stress-mediated Fas induction

The role of CaMKII in ER stress-induced Fas induction would predict that the kinase plays a critical role in ER stress-induced apoptosis. In a previous study, we showed that KN93 blocked cholesterol-induced macrophage apoptosis (25). Our goal here was to substantiate this important finding through genetic targeting of CaMKII $\gamma$. First, we used the active siRNA species described above and found that knockdown of CaMKII $\gamma$ suppressed cholesterol-induced apoptosis (Figure 3D). Second, cultured peritoneal macrophages derived from Camk2 $\mathrm{g}^{-/-}$mice showed no detectable CaMKII $\gamma$ protein by immunoblot (below). We found that cholesterol-induced apoptosis was suppressed in CaMKII $\gamma$-deficient macrophages to a degree similar to that found using the siRNA approach (Figure 3E).

To broaden the implications of our findings, we tested the role of CaMKII in ER stress-induced apoptosis using 2 additional models. The endothelium of advanced atheromata displays markers of UPR activation (17). Using thapsigargin-treated human aortic endothelial cells as a model, we showed that apoptosis under these conditions was markedly suppressed by KN93, but not by KN92 (Figure 3F). In a completely different model, we examined apoptosis of the neuronal cell line PC12 by 6-hydroxydopamine (6-OHDA), a model of Parkinson disease that has previously been shown to be mediated through activation of the UPR (33). We found that 6-OHDA decreased the survival of PC12 cells, and this effect was prevented by about $50 \%$ by pretreatment with AIP-II $(P<0.01$; Figure 3G). KN93 also suppressed 6-OHDA-induced apoptosis in PC12 cells (data not shown). These data show that CaMKII is involved in ER stress-induced apoptosis in a number of different scenarios.

JNK mediates the proapoptotic role of CaMKII in ER-stressed macrophages. Reports in the literature have suggested links between CaMKII and JNK $(34,35)$, and between JNK and Fas induction $(36,37)$. Moreover, we reported that JNK is activated by and necessary for apoptosis in ER stress-induced macrophages (15). We therefore hypothesized the presence of a CaMKII-JNK-Fas pathway. To determine a possible role for JNK in cholesterol-mediated Fas induction, macrophages were loaded with cholesterol in the absence or presence of the JNK inhibitor SP600125. The JNK inhibitor prevented the induction of Fas $\mathrm{mRNA}$ and protein in the cholesterol-loaded cells (Figure 4, A and B). To confirm these results, macrophages from WT mice and from mice lacking JNK2, the predominant form expressed in macrophages (15), were loaded with cholesterol and assayed for Fas mRNA and protein. Fas induc- 

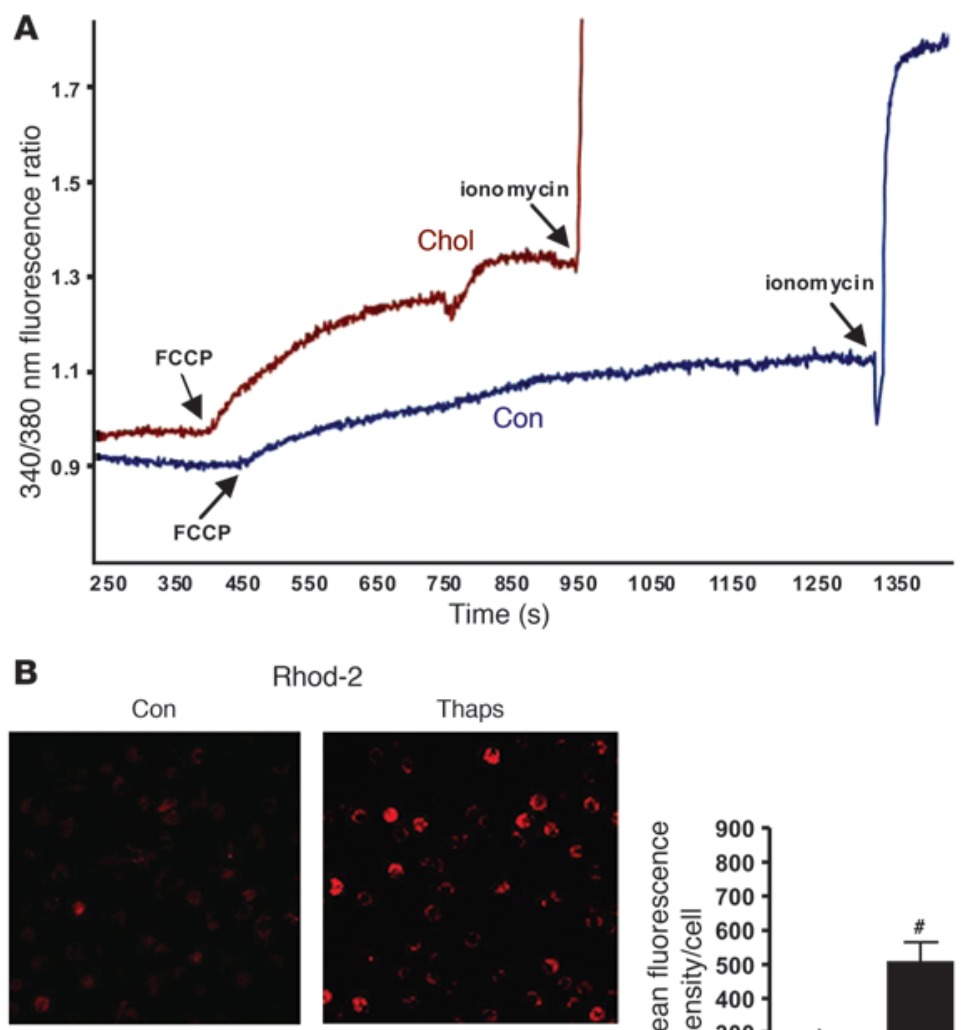

Chol $5 \mathrm{~h}$

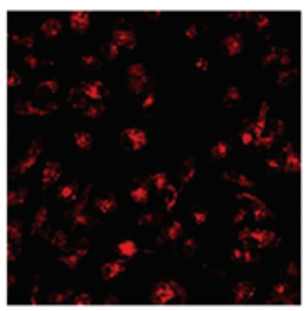

Rhod-2

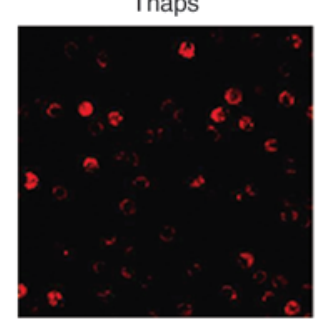

Chol $7 \mathrm{~h}$

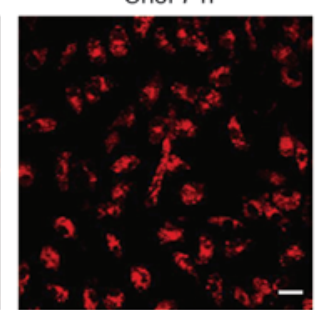

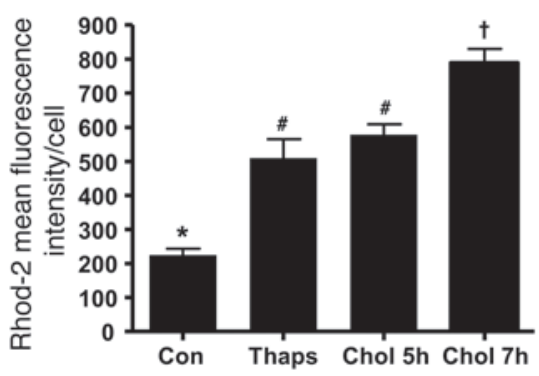

tion was markedly suppressed in Jnk2-/- macrophages (Figure 4, C and D). Next, we determined whether CaMKII was upstream of JNK activation. Inhibition of CaMKII in cholesterol-loaded macrophages by AIP-II blocked JNK phosphorylation (Figure 4E). Similar results were found when CaMKII was inhibited by KN93 (data not shown). AIP-II did not inhibit LPS-induced JNK phosphorylation (Figure 4F), indicating that AIP-II is not simply a general inhibitor of JNK activation. In considering possible mechanisms through which CaMKII might activate JNK, we probed the upstream JNK kinase mitogen-activated protein kinase kinase-4 (MKK4) for CaMKII-dependent phosphorylation. Cholesterol loading was associated with an increase in the phosphorylation of MKK4, and phosphorylation was markedly suppressed by treatment of the cells with AIP-II (Figure 4E). The upstream kinase MKK7 was not phosphorylated by cholesterol loading of macrophages (data not shown). Cultured peritoneal macrophages derived from Camk $2 g^{-/}$mice, which showed no detectable CaMKII $\gamma$ protein by immunoblot, also responded to cholesterol loading with substantially less induction of Fas, phospho-JNK, and phospho-MKK4 than macrophages from WT mice (Figure 4G). In summary, the combined data in Figures 1-4 support a model in which ER stress triggers a critical apoptosis pathway involving CaMKII activation,
Figure 6

ER stress in macrophages leads to the accumulation of calcium in the mitochondria. (A) Macrophages were plated on 25-mm coverslips and grown to confluency. The cells were incubated under control or cholesterol-loading conditions for 1 hour and stained with 5 uM Fura-2 for 30 minutes. The cells were then rinsed in medium and examined by fluorescence microscopy, where calcium fluorescence was measured every second. FCCP $(1 \mu \mathrm{M})$, a mitochondrial uncoupler, and ionomycin $(10 \mu \mathrm{M})$ were added at the indicated times to release mitochondrial and overall intracellular calcium stores, respectively. (B) Macrophages were treated with medium alone as control, with $0.25 \mu \mathrm{M}$ thapsigargin for 1 hour, or under cholesterol-loading conditions for 2 or 4 hours. At the end of each incubation, $10 \mu \mathrm{M}$ Rhod-2, a mitochondrial-specific fluorescent calcium dye, was added to the media, and the cells were incubated on ice for 1 hour at $4^{\circ} \mathrm{C}$. The cells were then washed and incubated for an additional 5 hours with thapsigargin or under cholesterol-loading conditions, such that total incubation time for thapsigargin was 5 hours and for cholesterol 5 or 7 hours. At the end of the incubation period, the cells were visualized using confocal microscopy and imaged as described in Methods. Scale bar: $10 \mu \mathrm{m}$. Fluorescence intensity for approximately 100 cells was measured for each treatment group. Differing symbols indicate $P<0.01$; identical symbols indicate differences that are not significant. which, through activation of JNK, and perhaps via activation of MKK4, induces proapoptotic Fas.

Release of mitochondrial cytochrome c and loss of mitochondrial membrane potential in cholesterol-loaded macrophages is CaMKII dependent. We have previously shown that cholesterol loading leads to release of cytochrome $\mathrm{c}$ and induction of caspase 9-mediated apoptosis as well as loss of mitochondrial membrane potential $\left(\Delta \psi_{\mathrm{m}}\right)(11)$. While the exact relationship between cytochrome $\mathrm{c}$ release, which is caused by outer mitochondrial membrane permeabilization, and $\Delta \psi_{\mathrm{m}}$ is controversial and likely varies in different apoptotic scenarios, both events have been associated with apoptosis (38). In the context of the current study, we sought to determine whether there was a link between CaMKII signaling and these events in ER-stressed macrophages. We first verified that a key event in mitochondrial-mediated apoptosis, namely, $\Delta \psi_{\mathrm{m}}$, was present in the current serum-replete model of cholesterol-induced apoptosis. There was marked loss of MitoTracker Red staining, a marker of mitochondrial membrane potential, after 12 and 16 hours of cholesterol loading (Figure 5A). The loss of MitoTracker Red staining was prevented by treatment with KN93, but not by KN92 treatment (Figure 5A). Similar data were obtained when macrophages from Camg $2 \mathrm{~g}^{-1-}$ mice were used instead of treating WT macrophages with KN93: the cholesterol- 
induced decrease in MitoTracker Red staining was much greater in WT than in CaMKII $\gamma$-deficient macrophages (Figure 5B).

We next turned our attention to cytochrome $c$ release. Cholesterol loading led to a loss of cytochrome $\mathrm{c}$ in the mitochondria and appearance in the cytosol (Figure 5C). This event was suppressed by KN93, but not by KN92 (Figure 5C). Note that the mitochondria were free of cytosolic contamination, as assessed by the absence of tubulin (Figure 5C) and of $\beta$-actin and GAPDH (data not shown). Thus, CaMKII signaling is necessary for $\Delta \psi_{\mathrm{m}}$ and mitochondrial cytochrome c release during cholesterol-induced ER stress.

We recently showed that the proapoptotic signal transducer STAT1 was activated downstream of CaMKII in ER-stressed macrophages and was required for ER stress-induced macrophage apoptosis in vitro and for advanced lesional macrophage apoptosis and plaque necrosis in vivo (25). Moreover, in other systems, STAT1-induced apoptosis has been linked to the $\Delta \psi_{\mathrm{m}}(39)$. To determine whether CaMKII-mediated $\Delta \psi_{\mathrm{m}}$ was STAT1 dependent, we assayed MitoTracker Red staining in cholesterol-loaded macrophages from WT versus Stat $1^{-/-}$mice. Cholesterol-induced loss of MitoTracker Red staining was completely unaffected by STAT1 deficiency (Figure 5D). We next considered the possibility that JNK might link CaMKII activation with $\Delta \psi_{\mathrm{m}}$, as it did in Fas induction (see above). However, inhibition of JNK did not block the decrease in MitoTracker Red staining in cholesterol-loaded macrophages (Figure 5E). Thus, CAMKII-mediated $\Delta \psi_{\mathrm{m}}$ in ER-stressed macrophages involves a mechanism that is independent of both STAT1 and JNK signaling.

ER stress results in CaMKII-dependent accumulation of calcium in the mitochondria. A potential mechanism linking ER stress with proapoptotic mitochondrial dysfunction is excess transfer of calcium from ER stores into the mitochondria, leading to $\Delta \psi_{\mathrm{m}}(40-42)$. To determine whether mitochondrial calcium uptake is increased in ER-stressed macrophages, macrophages were incubated under cholesterol-loading conditions for 1.5 hours, the last 30 minutes of which included loading the cells with the cytoplasmic fluorescent calcium sensor Fura-2. At the end of the incubation period, the cells were imaged for Fura-2 fluorescence and exposed to the uncoupling agent FCCP, which releases mitochondrial calcium into the cytoplasm. Thus, the post-FCCP increase in Fura-2 fluorescence is proportional to the content of mitochondrial calcium at the end of the cholesterol loading period (43). The fluorescence intensity of Fura-2 after FCCP treatment was substantially greater in cholesterol-loaded than in control macrophages (Figure 6A). As a control, we showed that cells under both conditions responded similarly to ionomycin-induced expansion of cytosolic calcium pools. This experiment was conducted on several fields of cells with similar results (data not shown). These data indicate that mitochondrial calcium content was increased by cholesterol loading.

To corroborate our finding that mitochondrial calcium was increased by cholesterol, we assayed mitochondrial calcium by an alternative, direct method. We loaded cells with the calcium-sensitive fluorescent dye Rhod-2 under conditions in which the dye is sequestered in mitochondria (44). Rhod-2 has a net positive charge and therefore preferentially accumulates in the mitochondria. Moreover, the dye is loaded into the cells at $4^{\circ} \mathrm{C}$ to prevent cleavage of the ester as it traffics to the mitochondria, followed by incubation at $37^{\circ} \mathrm{C}$ to allow mitochondrial esterases to activate the dye (44). Both the ER stressor thapsigargin and cholesterol loading caused a marked increase in Rhod-2 fluorescence in macrophages (Figure 6B). Note that addition of type A scavenger receptor (SRA) ligands to thapsigargin, which is necessary for apoptosis, did not increase Rhod-2 fluorescence further (data not shown), consistent with the notion that the ER stress component of lipoprotein-cholesterol loading, not the PRR component, is responsible for the increase in mitochondrial calcium. In additional experiments, when Rhod-2-labeled macrophages were loaded with cholesterol for a long enough period of time to induce apoptosis, the apoptotic cells were entirely represented among the population of cells that were Rhod-2-positive (data not shown).

We next determined whether the increase in mitochondrial calcium was dependent on CaMKII. KN93, but not KN92, caused a marked decrease in Rhod-2 fluorescence in cholesterol-loaded macrophages (Figure 7A). Cells treated with the mitochondrial calcium uniporter inhibitor RU360 (45) served as a positive control for loss of mitochondrial Rhod-2 fluorescence. Consistent with the idea that these mitochondrial calcium processes were directly related to ER stress (see above), similar data were obtained using thapsigargin-treated macrophages (Figure 7B).

The role of CaMKII in mitochondrial membrane depolarization and mitochondrial calcium uptake in ER-stressed macrophages could involve a CaMKII-dependent process occurring at the mitochondrial membrane. If so, we might expect to see an increase in mitochondria-associated CaMKII upon ER stress induction. For example, activation of CaMKII $\alpha$ in neurons leads to its translocation from the cytoplasm to postsynaptic membrane receptors (46). To test this idea, cytosol-free mitochondria were probed for total and phosphorylated CaMKII under control and cholesterol-loading conditions. There was a marked increase in mitochondria-associated CaMKII after 6 hours of cholesterol loading, and mitochondria also showed an increase in phospho-CaMKII under these conditions (Figure 7C; purity of the mitochondrial fraction is shown by the bottom immunoblot). These data raise the possibility that cholesterol loading, and perhaps ER stress in general, causes a translocation of CaMKII from the cytoplasm to the mitochondria.

Finally, we sought to show the predicted causal links among mitochondrial calcium uptake, $\Delta \psi_{\mathrm{m}}$, and apoptosis in the ER stress/PRR model. RU360 blocked the cholesterol-induced loss of MitoTracker Red staining (Figure 7D), indicating the role of mitochondrial calcium uptake in ER stress-induced $\Delta \psi_{\mathrm{m}}$. Most importantly, ER stress/PRR-induced apoptosis was suppressed by RU360 (Figure 7E). Thus, mitochondrial calcium uptake is induced by ER stress in a CaMKII-dependent manner, and these events are important for ER stress-induced apoptosis.

Importance of CaMKII in macrophage apoptosis and $\Delta \psi_{m}$ in ER-stressed mice. To test the role of CaMKII $\gamma$ in macrophage apoptosis in vivo, we took advantage of a model of systemic ER stress in which mice are injected i.v. with tunicamycin (47). We treated WT and Camk $2 \mathrm{~g}^{-1-}$ mice with tunicamycin, and, 24 hours later, harvested peritoneal macrophages and assayed them for CaMKII $\gamma$ protein, apoptosis, and mitochondrial membrane potential. As above, CaMKII protein was present in macrophages from WT mice but was undetectable in macrophages from Camk2 $\mathrm{g}^{-/-}$mice (Figure 8A). Macrophages from the tunicamycin-treated WT mice showed a marked increase in apoptosis compared with vehicle-treated WT mice, and this increment was significantly blunted in macrophages from tunicamycintreated Camk $2 \mathrm{~g}^{-1-}$ mice (Figure 8B). Similarly, tunicamycin treatment of WT mice led to $\Delta \psi_{\mathrm{m}}$, which was not seen in macrophages from tunicamycin-treated Camk2 $\mathrm{g}^{-1-}$ mice (Figure 8C). Another experiment using i.p. tunicamycin was conducted to examine apop- 
A

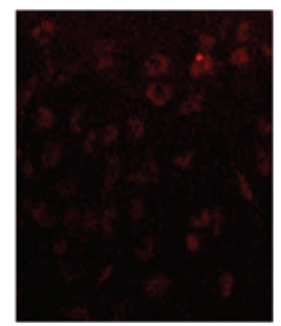

Chol KN93

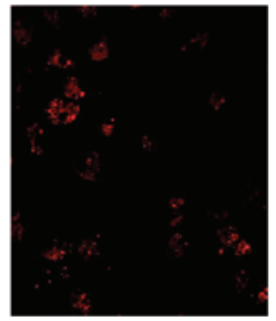

B

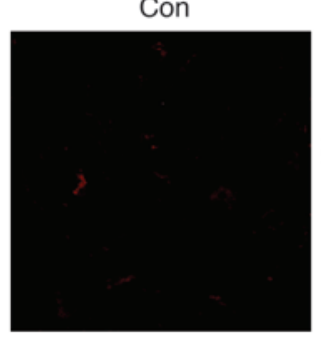

Thaps KN93

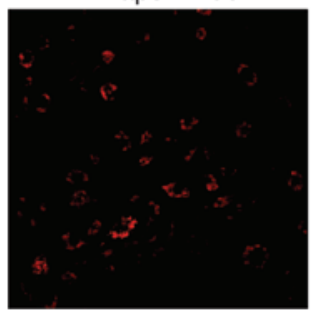

C

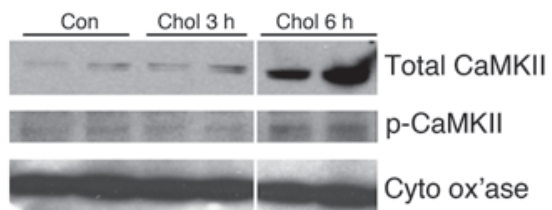

Isolated mitochondria

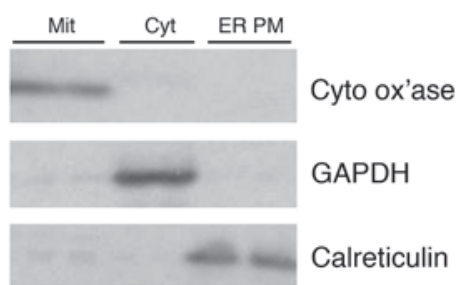

Chol

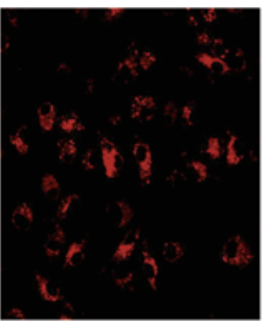

Chol KN92

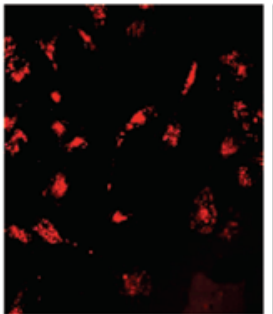

Thaps

Thaps KN92
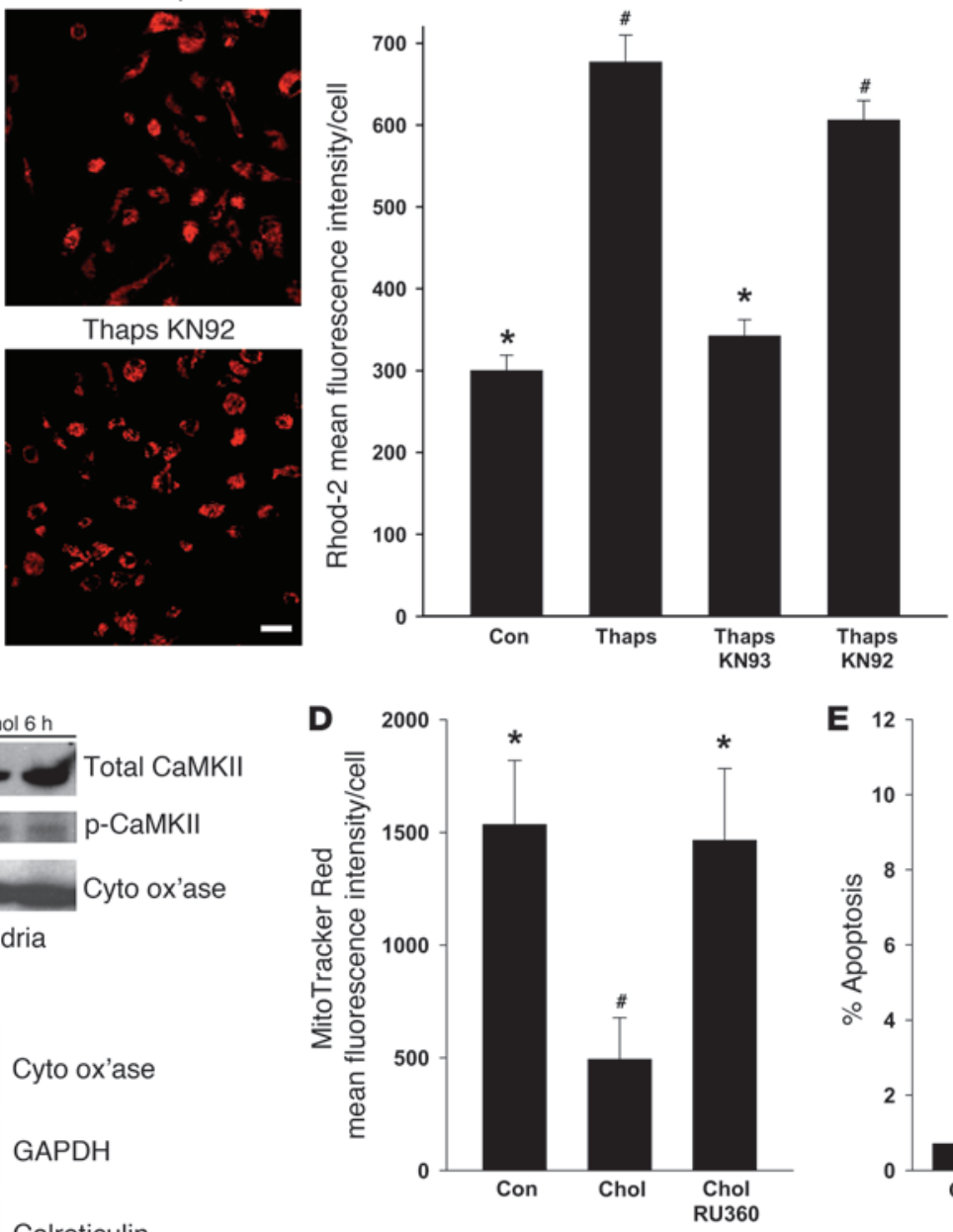

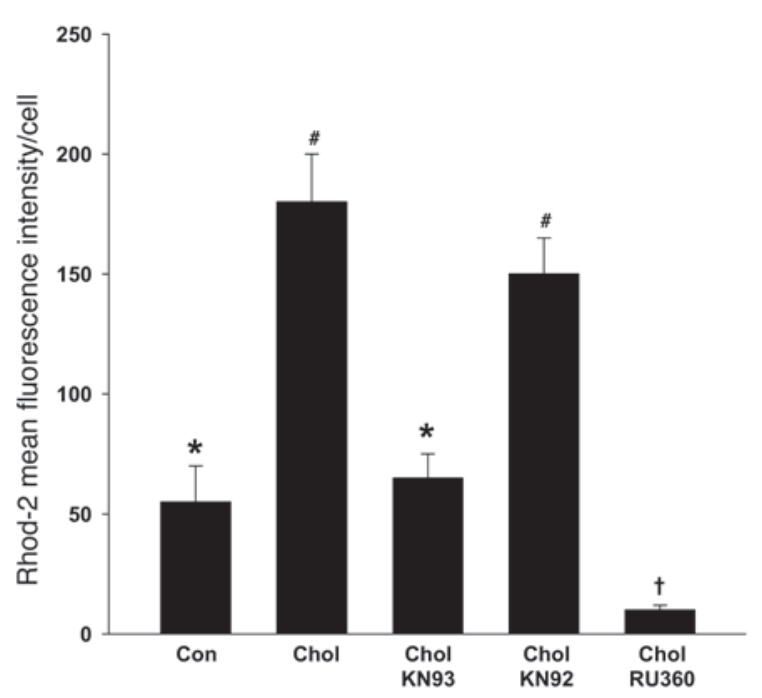

E 12

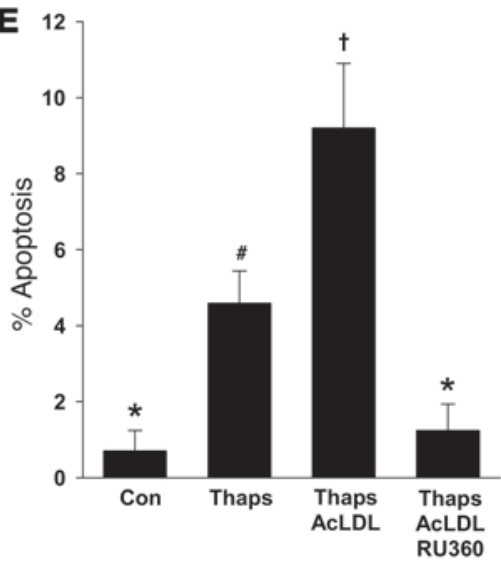




\section{Figure 7}

ER stress-induced mitochondrial calcium uptake is dependent on CaMKII. (A and B) Macrophages were incubated under control or cholesterol-loading conditions (A) or with $0.25 \mathrm{mM}$ thapsigargin as the ER

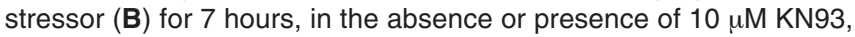
KN92, or RU360 after 1 hour pretreatment. Rhod-2 labeling and quantification were then carried out as described in Figure 5. Scale bars: $10 \mu \mathrm{m}$. (C) Macrophages were incubated under control or cholesterolloading conditions for the indicated times. Cytosol-free mitochondria were isolated and immunoblotted for total CaMKII, phospho-CaMKII, and cytochrome c oxidase (Cyto ox'ase). Mitochondria (Mit), cytosol (Cyt), and ER-plasma membrane (ER PM) fractions - immunoblotted for the mitochondrial marker cytochrome $c$ oxidase, the cytosol marker GAPDH, and the ER marker calreticulin - are also shown. (D) Macrophages were incubated under control or cholesterol-loading conditions for 12 hours with or without $10 \mu \mathrm{M}$ RU360 after 1 hour pretreatment, and then stained with MitoTracker Red. (E) Macrophages were incubated for 20 hours with medium alone as control or with medium containing $0.25 \mu \mathrm{M}$ thapsigargin; thapsigargin and $50 \mu \mathrm{g} / \mathrm{ml}$ acetylLDL (AcLDL); or thapsigargin, acetyl-LDL, and RU360. Apoptosis was then assayed quantified. Differing symbols indicate $P<0.01$; identical symbols indicate differences that are not significant.

tosis in splenic macrophages in situ (Figure 8D). Tunicamycin led to a marked increase in apoptosis in CD68-positive macrophages, as well as other cells, in the spleen, and this effect was completely abrogated in Camk $2 \mathrm{~g}^{-/}$mice. Thus, paralleling the data in vitro, CaMKII $\gamma$ plays an important role in macrophage apoptosis and $\Delta \psi_{\mathrm{m}}$ induced by systemic ER stress in vivo.

The renal tubular epithelium is highly responsive to ER stress inducers, and ER stress in these cells is associated with renal dysfunction and is a cellular pathological end point of a number of renal diseases $(47,48)$. To test the application of our model to the kidney and assess functional significance in vivo, we used the tunicamycin mouse model described above. Using RT-PCR, we found that the major isoform of CaMKII expressed in murine kidneys was CaMKII $\gamma$ (data not shown). Kidneys harvested from tunicamycin-treated WT mice show extensive apoptosis in renal tubular epithelium (Figure 9A), consistent with previous findings (47). Apoptosis in these cells was markedly suppressed in tunicamycin-treated Camk $2 \mathrm{~g}^{-1-}$ mice. We next examined renal function in this model. Two measures of renal function, serum creatinine and urine albumin/creatinine ratio, were abnormal in tunicamycintreated WT mice but were within normal range in Camk2 $\mathrm{g}^{-1-}$ mice (Figure 9B). These data indicate that CaMKII $\gamma$ has a more general role in ER stress-induced apoptosis and organ function in vivo.

\section{Discussion}

ER stress-induced apoptosis is a key pathogenic event in disease processes as divergent as diabetes, cancer, renal disease, heart disease, and neurological diseases $(5-8,21,48)$. The overall objective of this study was to gain new information related to the mechanism of ER stress-induced apoptosis, with the specific goal of linking upstream ER stress-mediated events to downstream apoptosis execution pathways in a model not involving caspase- 12 (22). Our conclusions are depicted in the model in Figure 10. CaMKII is activated by cytosolic calcium resulting from ER stressinduced release of ER calcium stores (25). Given the role of CHOP in our and other models of ER stress-induced apoptosis $(20,47$, 49-52), we are currently interested in the concept that prolonged CHOP expression leads to the release of ER calcium stores (26,
41). CaMKII activity is likely sustained through autophosphorylation and possibly oxidation of the autoinhibitory domain, but it is also possible that inhibition of CaMKII phosphatase (53) may also contribute to sustained activation. Activated CaMKII then enables apoptosis through at least 3 pathways, all of which are necessary for cell death: (a) activation of JNK, which induces Fas; (b) stimulation of calcium uptake by the mitochondria, leading to outer mitochondrial membrane permeabilization and release of apoptogens as well as $\Delta \psi_{\mathrm{m}}$; and (c) activation of STAT1, which has previously been shown to promote apoptosis in diverse cell types by a number of transcriptional and perhaps nontranscriptional mechanisms (54). Although studies in other cells have shown that proapoptotic mitochondrial dysfunction can be triggered by the mitochondrial uptake of ER-released calcium in the setting of ER stress (41) and that CaMKII can play roles in apoptosis (55-59), the current study is the first to our knowledge to link these pathways into an integrated model of apoptosis and to show that CaMKII is essential for mitochondrial calcium uptake. Another important point relates to our finding that neither induction of Fas (data not shown) nor mitochondrial depolarization was dependent on STAT1. Together with our previous publication showing that activated STAT1 is necessary for apoptosis (25), these data imply that the action of CaMKII on Fas and mitochondria are not sufficient for apoptosis in the absence of STAT1. This concept fits well with our overall concept that individual proapoptotic hits are subthreshold (15) and that multiple subthreshold hits - in this case, all 3 of the CaMKII pathways depicted in Figure 10 - are necessary for apoptosis. In addition to the activation of multiple subthreshold proapoptotic pathways, the multihit concept also involves suppression of compensatory cell survival pathways, such as those involving IFN- $\beta$ and Akt-p38 $\alpha$, that are often induced in parallel with UPR activation $(23,60)$.

The data in this report as well as ongoing work in our laboratory have suggested that the role of CaMKII in ER stress-induced apoptosis may be part of a positive feedback amplification loop. ERstressed macrophages have heightened ROS (G. Li, T.A. Seimon, and I. Tabas, unpublished observations), possibly related to increased mitochondrial calcium influx, and ROS might lead to sustained calcium-independent CaMKII activity through Met281/282 oxidation in the autoinhibitory domain of the enzyme (Figure $2 \mathrm{G}$ and ref. 32). ROS may also contribute to this pathway by further stimulating ER calcium release through activation of inositolphosphate receptors (61). Moreover, the calcium that accumulates in mitochondria is likely released from those mitochondria that first become dysfunctional, and this increase in cytosolic calcium might further amplify the CaMKII signal (62). The fact that both the Fas and mitochondrial pathways of apoptosis require CaMKII provides a plausible explanation for why blocking mitochondrial uptake with RU360 almost completely inhibited apoptosis (Figure $7 \mathrm{E})$. In particular, breaking the putative positive feedback loop at the level of mitochondrial calcium uptake might ultimately lead to decreased activation of CaMKII and thus decrease induction of Fas, thereby shutting down both the mitochondrial and the Fas pathways of apoptosis. We are seeking further evidence for this concept in ongoing experiments. Regarding the role of CaMKII in mitochondrial calcium uptake, further mechanistic work will need to distinguish between 2 fundamental processes that could be affected by the kinase: promotion of physical contact between the ER and mitochondria, an important and regulated step in ERto-mitochondrial calcium transfer (63); versus enhancement of cal- 
A
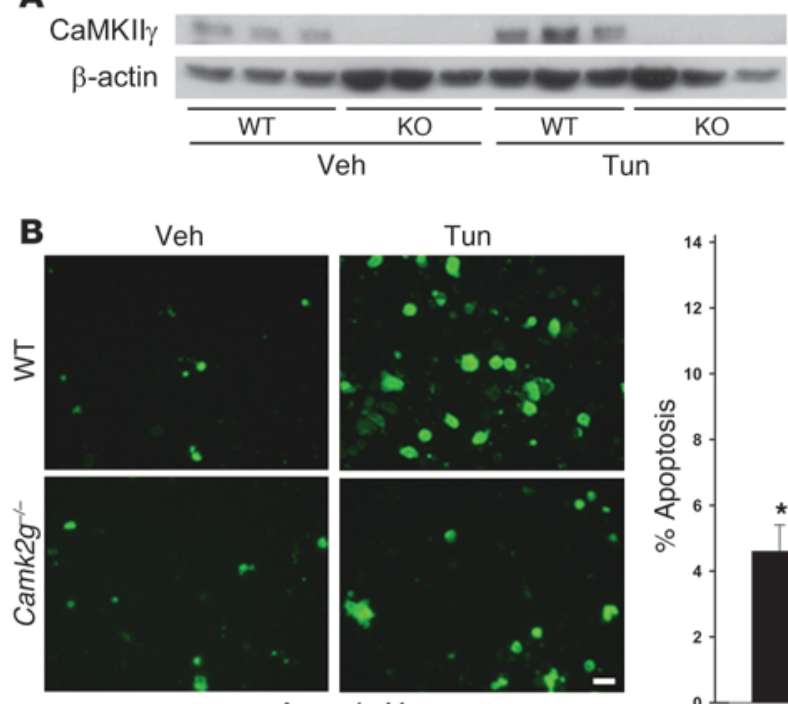

Annexin V
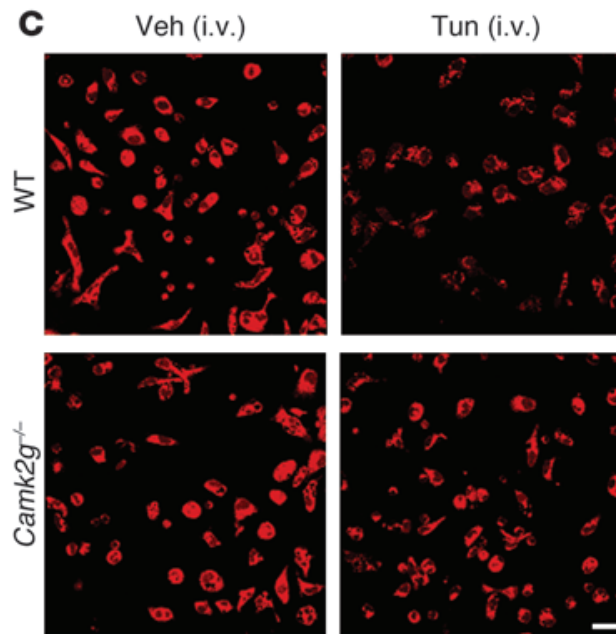

MitoTracker Red

D
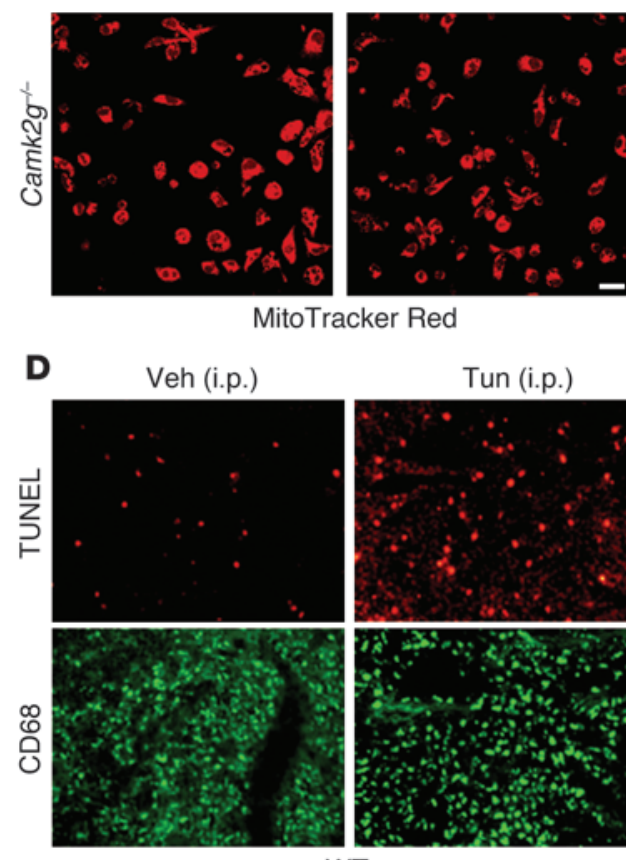

Tun (ip.)

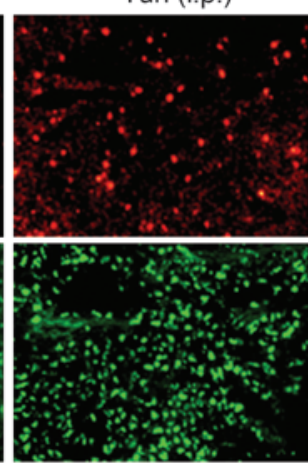

WT
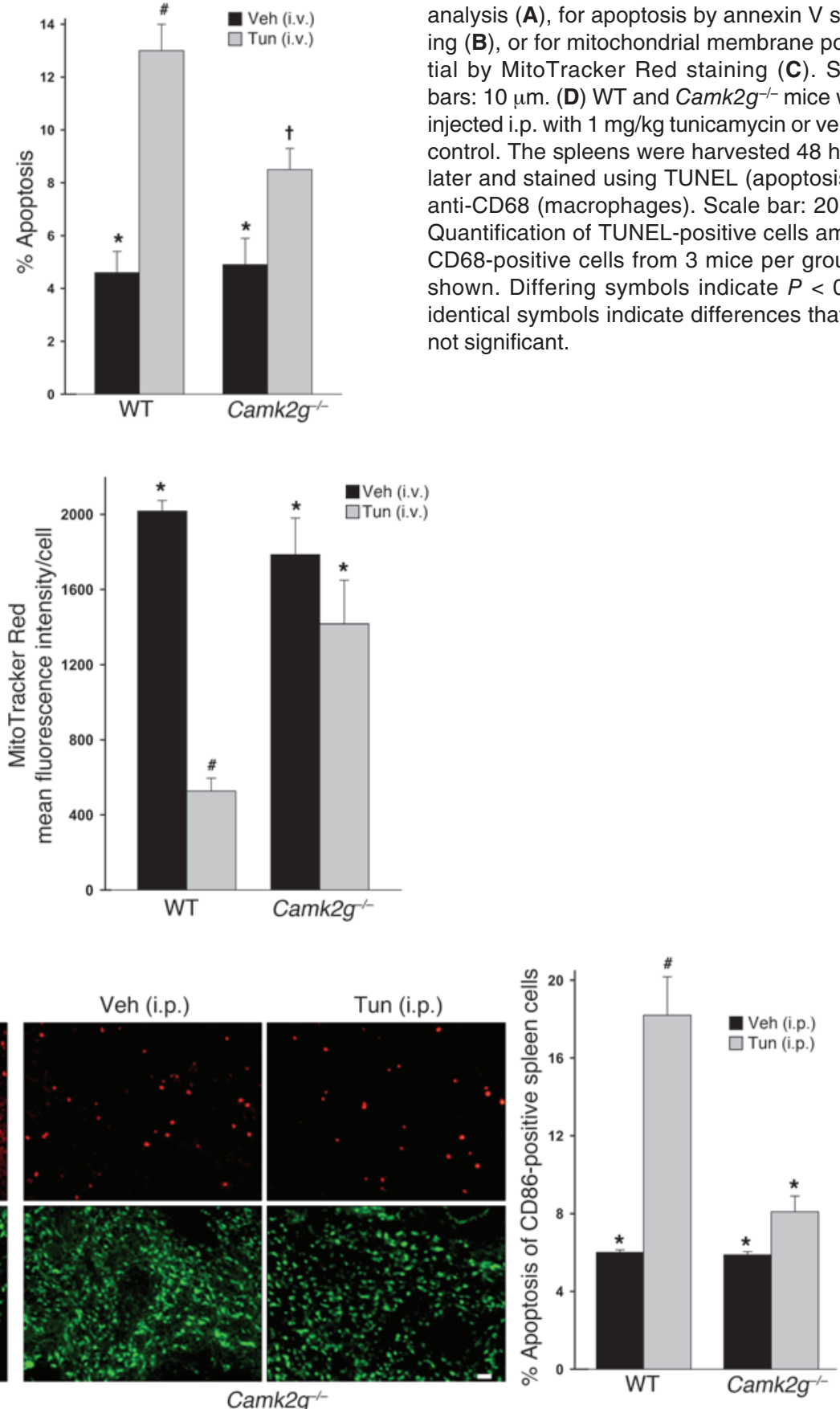

cium transport into the mitochondria per se, for example, through direct or indirect effects on the mitochondrial calcium uniporter (45). With respect to the latter possibility, other calcium transporters, pores, and transcriptional regulators are known CaMKII substrates, including the L-type calcium channel $(64,65)$, the ryanodine receptor $(66,67)$, and histone deacetylase $4(68)$.

\section{Figure 8}

Importance of CaMKIly in macrophage apoptosis and $\Delta \psi_{m}$ in ER-stressed mice. (A-C) WT and Camk2 $\mathrm{g}^{-/-}$mice were injected i.v. with tunicamycin or vehicle control. Peritoneal macrophages were harvested 24 hours later and then assayed for CaMKIl $\gamma$ protein by immunoblot analysis (A), for apoptosis by annexin $V$ staining (B), or for mitochondrial membrane potential by MitoTracker Red staining (C). Scale bars: $10 \mu \mathrm{m}$. (D) WT and Camk2 $\mathrm{g}^{-/-}$mice were injected i.p. with $1 \mathrm{mg} / \mathrm{kg}$ tunicamycin or vehicle control. The spleens were harvested 48 hours later and stained using TUNEL (apoptosis) or anti-CD68 (macrophages). Scale bar: $20 \mu \mathrm{m}$. Quantification of TUNEL-positive cells among CD68-positive cells from 3 mice per group is shown. Differing symbols indicate $P<0.01$; identical symbols indicate differences that are not significant. 
A

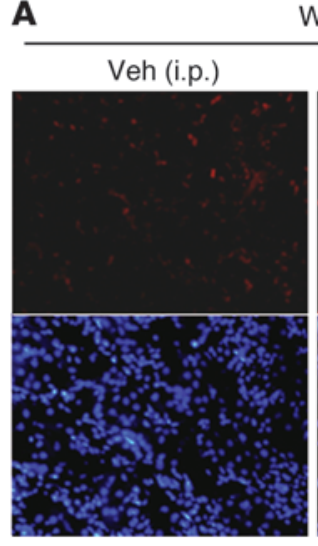

WT

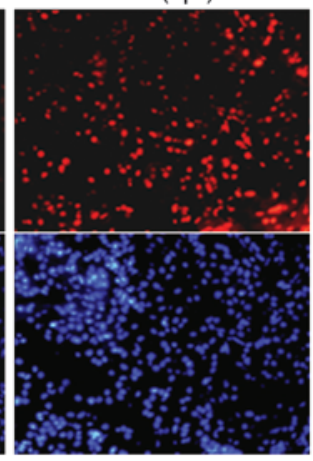

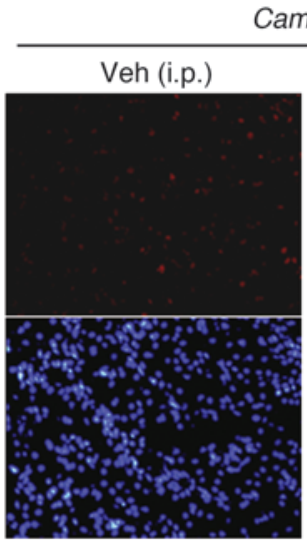

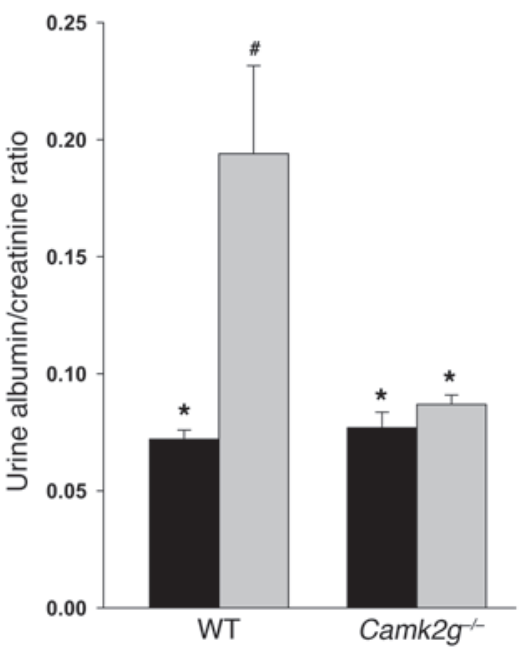

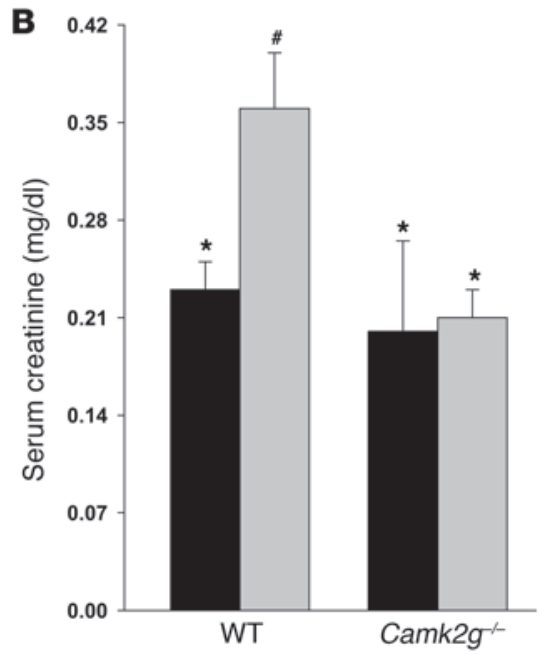

Camk2g ${ }^{-}$
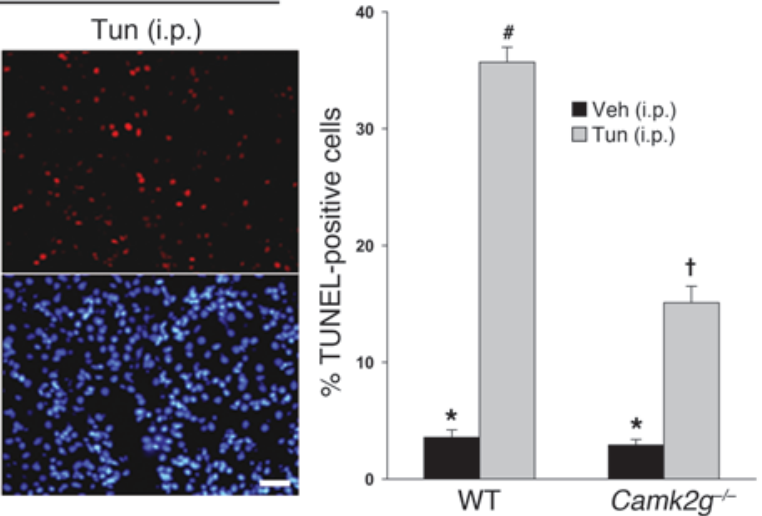

Figure 9

Importance of CaMKIly in renal tubular epithelial cell apoptosis and renal function in ER-stressed mice. WT and Camk2g $\mathrm{g}^{-/-}$mice were injected i.p. with $1 \mathrm{mg} / \mathrm{kg}$ tunicamycin or vehicle control. (A) The kidneys were harvested 48 hours later and stained using TUNEL (apoptosis) or DAPI (nuclei). Scale bar: $20 \mu \mathrm{m}$. Quantification of TUNEL-positive cells from 3 mice per group is shown. (B) Serum creatinine levels and urine albumin levels (normalized to urine creatinine) were determined for all groups of mice. Differing symbols indicate $P<0.01$ (A) or $P<0.05$ (B); identical symbols indicate differences that are not significant.

quent plaque necrosis $(13,19,20)$. Moreover, the proinflammatory effects of cell death in the setting of defective efferocytosis may also contribute to plaque progression (9). In addition, preapoptotic ER stress itself has proinflammatory effects in macrophages through activation of both a JNK-TNF- $\alpha$ pathway and a CHOP-ERK-IL-6 pathway (69). In preliminary experiments, we found that TNF- $\alpha$ secretion was suppressed in cholesterol-loaded macrophages from Camk $2 g^{-/-}$versus WT mice (our unpublished observations), which is consistent with the role of CaMKII in JNK activation. In contrast, the ERK-IL-6 pathway was not suppressed in CaMKIIdeficient cells, which suggests that CHOP activates ERK through a pathway not involving CaMKII. Although further work will be needed to fully sort out the role and mechanisms of CaMKII in the inflammatory responses of ER-stressed macrophages, the apoptosis findings in this report raise the possibility of a novel therapeutic approach to the prevention of plaque disruption and acute cardiovascular events, namely through inhibition of CaMKII. CaMKII inhibitors have been tested successfully in animal models in other scenarios in which CaMKII is thought to contribute to disease pro- gression $(70,71)$. In the case of targeting macrophages, increased specificity could be gained by targeting the non-neuronal $\gamma$ isoform of CaMKII. In a broader sense, we have shown - for the first time to our knowledge - that CaMKII is involved in ER stress-induced endothelial cell apoptosis, another event that may be important in atherosclerosis $(17,72)$; in 6-OHDA-induced death of neuronal cells, a process thought to be important in Parkinson disease (33); and in ER stress-induced renal epithelial cell apoptosis and renal dysfunction, an end point of a number of renal pathologies (48). Thus, CaMKII inhibition and other strategies targeting specific mechanisms linking ER stress to downstream death pathways may offer therapeutic approaches to a variety of disease processes in which ER stress-induced apoptosis plays a pathologic role.

\section{Methods}

Reagents. Cell culture media and reagents were from Invitrogen. HPLCgrade organic solvents were from Fisher Scientific. LDL (d, 1.020-1.063) was isolated from fresh human plasma by preparative ultracentrifugation as described previously (73). Our studies with purchased human plasma 


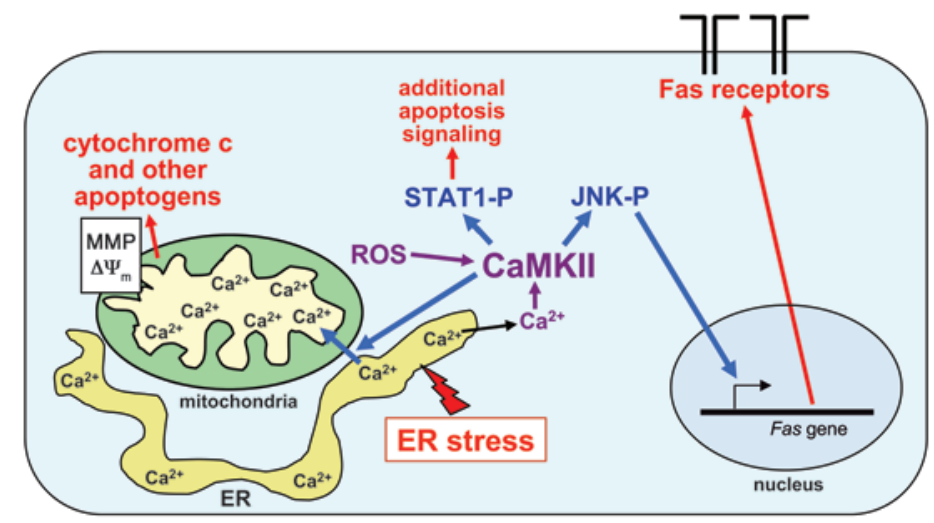

\section{Figure 10}

Schematic of calcium-CaMKII-mediated events leading to ER stress-induced macrophage apoptosis. ER stress depletes the calcium stores within the ER lumen. Calcium subsequently accumulates in the cytoplasm and activates CaMKII. CaMKII activation, which is sustained through autophosphorylation and possibly ROS-mediated oxidation, enables apoptosis through at least 3 pathways: JNK-mediated Fas induction; promotion of mitochondrial calcium uptake, followed by mitochondrial membrane permeabilization (MMP), release of apoptogens, and $\Delta \psi_{\mathrm{m}} ;$ and activation of STAT1, a proapoptotic signal transducer. See text for details. and purchased human aortic endothelial cells were exempt from IRB approval because the sources were anonymous. Acetyl-LDL was prepared by reaction of LDL with acetic anhydride (74). U18666A, KN93, KN92, AIP-II, SP6000125, and RU360 were from Calbiochem. All other chemical reagents, including the ACAT inhibitor 58035 (3-[decyldimethylsilyl]- $N$-[2(4-methylphenyl)-1-phenylethyl] propanamide), were from Sigma-Aldrich unless otherwise specified.

Mice. C57BL/6J and Fas-deficient ( $L p r$ ) mice used for peritoneal macrophage isolation were purchased from The Jackson Laboratory. Jnk2-- mice were provided by R. Flavell (Yale University School of Medicine, New Haven, Connecticut, USA; ref. 75), and Stat $1^{-/-}$mice were provided by D. Levy (New York University School of Medicine, New York, New York, USA; ref. 76). $\mathrm{Balb} / \mathrm{c}$ mice from Taconic were used as WT controls for the Stat $1^{-/-}$experiments. Camk2 $g^{-1-}$ mice were made by first generating mice in which exons 1 and 2 of the Camk2 $\mathrm{g}^{-/}$locus were flanked by loxP sites. These 2 exons encode part of the catalytic domain of CaMKII $\gamma$, including the ATP binding motif that is essential for kinase function. Because of the similar genomic organization, the targeting strategy was similar to the generation of CaMKII $\delta$-null mice (68). To delete exons 1 and 2 in the germline, Camk2 $g^{l}$ mice were crossed with CAG-Cre transgenic mice, in which the Cre gene is under control of the cytomegalovirus immediate early enhancer-chicken $\beta$-actin hybrid (CAG) promoter (77). The resulting Camk2 $\mathrm{g}^{+/-}$mice were bred to yield Camk2 $\mathrm{g}^{-1}$ mice, which were then further bred into the C57BL/6J background. Correct gene targeting was confirmed by Southern blot, RT-PCR, and Western blot analysis: no alternative translation product containing the C-terminal part of CaMKII $\gamma$ was generated. Camk $2 \mathrm{~g}^{-1-}$ mice were viable and developed to adulthood without obvious morphological or functional abnormalities, except that female Camk2 $g^{--}$mice were infertile. Thus, Camk2 $g^{--}$mice were generated by breeding Camk $2 \mathrm{~g}^{+/-}$females with Camk2 $\mathrm{g}^{-/}$males. Some of the breeding occurred in the laboratory of R.M. Schultz and P. Stein (University of Pennsylvania, Philadelphia, Pennsylvania, USA), who provided mice for this study. All animal procedures used in this study were approved by the Institutional Animal Care and Use Committee of Columbia University.

Cells. Peritoneal macrophages from adult female mice were harvested 3 days after i.p. injection of concanavalin A or 4 days after i.p. injection of methyl-BSA in mice that had previously been immunized with this antigen (69). Macrophages were harvested by peritoneal lavage with ice-cold PBS and maintained in DMEM containing 10\% FBS and 20\% L cell-conditioned medium. The medium was replaced every 24 hours until the cells reached $80 \%-90 \%$ confluency. Cholesterol loading was achieved by incubating the cells with $50 \mu \mathrm{g} / \mathrm{ml}$ acetyl-LDL plus $10 \mu \mathrm{g} / \mathrm{ml} 58035$ for the indicated times (15). Cholesteryl ester loading was achieved by using acetyl-LDL alone. For experiments in which the macrophages were incubated with KN93, KN92, or SP6000125, the vehicle control was DMSO; for experiments involving
BAPTA-AM, the vehicle control was 0.02\% F127 Pleuronic in DMSO. All chemical inhibitors were added to the cells 60 minutes prior to the beginning of the experiment and were also included in the subsequent incubations. Human aortic endothelial cells, purchased from Lonza (CC-2635), were provided by $\mathrm{N}$. Terasaka (laboratory of A. Tall, Columbia University). The cells were cultured in EGM-2 media and used at $80 \%$ confluency. Neuronal PC12 cells were cultured and treated with nerve growth factor (NGF) as described previously (78). In brief, the cells were cultured for 8-10 days in RPMI 1640 medium (Cellgro) supplemented with $1 \%$ horse serum, penicillin/streptomycin, and $50 \mathrm{ng} / \mathrm{ml}$ recombinant human NGF from Genentech. The medium was changed every other day and immediately before treatments.

Apoptosis assay. For macrophages and endothelial cells, mid-stage and late-stage apoptosis was assayed by annexin $V$ and propidium iodine staining, respectively, with Vybrant Apoptosis Assay Kit No. 2 (Invitrogen). In the experiments conducted in this study, greater than $90 \%$ of the cells were annexin $\mathrm{V}$-positive and propidium iodine-negative. At the end of the incubation time, the cells were gently washed once with PBS and then incubated for 15 minutes at room temperature with $110 \mu$ lannexin-binding buffer (25 mM HEPES; $140 \mathrm{mM} \mathrm{NaCl} 1 \mathrm{mM}$ EDTA, pH 7.4; and 0.1\% BSA) containing $10 \mu \mathrm{l}$ Alexa Fluor 488-conjugated annexin $V$ and $1 \mu \mathrm{l}$ of $100 \mu \mathrm{g} / \mathrm{ml}$ propidium iodine. The staining mixture was removed and replaced with $110 \mu \mathrm{l}$ binding buffer. The cells were viewed immediately at room temperature with an Olympus IX-70 inverted fluorescent microscope equipped with filters appropriate for fluorescein and rhodamine, and images were obtained with a Cool Snap charge-coupled device camera (RS Photometrics) equipped with imaging software from Roper Scientific. We photographed 3 fields of approximately 650 cells for each condition, and the number of annexin $\mathrm{V} /$ propidium iodine-positive cells in each field was counted and expressed as a percent of the total number of cells. For PC1 2 cells, the cells were pretreated for 1 hour with CaMKII inhibitors as indicated and then exposed to 6-OHDA for 24 hours. The 6-OHDA was prepared before use as a $10-\mathrm{mM}$ stock solution, which was then diluted in medium to the indicated final concentrations. After 6-OHDA treatment, the cells were lysed, and viable nuclei were counted under the microscope in a Neubauer chamber as described previously (78).

Immunoblotting. Cell extracts were electrophoresed on $4 \%-20 \%$ gradient SDS-PAGE gels (Invitrogen) and transferred to $0.22-\mu \mathrm{M}$ nitrocellulose membranes. The membranes were blocked for 1 hour at room temperature in Tris-buffered saline with $0.1 \%$ Tween 20 (TBST) containing $5 \%(\mathrm{w} / \mathrm{v})$ nonfat milk. They were then incubated with primary antibody in TBST containing $5 \%$ nonfat milk or BSA at $4{ }^{\circ} \mathrm{C}$ overnight, followed by incubation with the appropriate secondary antibody coupled to horseradish peroxidase. Proteins were detected by ECL chemiluminescence (Pierce). Antibodies to Fas and HSP-90 were purchased from Assay Design; antibodies to cytochrome c, 
phospho-Ser257/Thr261 MKK-4, and total JNK were from Cell Signaling; antibodies to prohibitin and phospho-Thr183/Tyr185 JNK1/2 were from Abcam; antibodies to $\beta_{1}$ integrin and tubulin were from Santa Cruz Biotechnology Inc.; anti- $\beta$-actin antibody was purchased from Chemicon; antiphospho-Thr287 CaMKII was from Novus; anti-total CaMKII antibody was purchased from Santa Cruz Biotechnology Inc.; and antiserum against Ox-CaMKII was raised and characterized as described previously (32).

Immunoprecipitation. Cells were lysed by a 5 -minute exposure to $1 \%$ Triton-X in $50 \mathrm{mM}$ HEPES, $150 \mathrm{mM} \mathrm{NaCl}, 10 \mathrm{mM} \mathrm{Na}$ pyrophosphate, $10 \mathrm{mM}$ EDTA, $10 \mathrm{mM}$ EGTA, $1 \mathrm{mM} \mathrm{Na}_{3} \mathrm{VO}_{4}, 50 \mathrm{mM} \mathrm{NaF}, 1 \mathrm{mM} \mathrm{PMSF}$, and $5 \mu \mathrm{g} /$ $\mathrm{ml}$ leupeptin. The lysate ( $200 \mu \mathrm{g}$ protein) was brought to a total volume of $1 \mathrm{ml}$ with lysis buffer containing $0.3-0.6 \mu \mathrm{g}$ antibody and $80 \mu \mathrm{l}$ Sepharose beads. The mixture was rotated in a $1.5-\mathrm{ml}$ microfuge tube at $4^{\circ} \mathrm{C}$ for 16 hours. Immune complexes were collected by centrifugation at $16,000 \mathrm{~g}$ and washed 3 times with chilled lysis buffer, followed by boiling in $2 \%$ Laemmli buffer for 5 minutes. The material was then subjected to SDS-PAGE and immunoblotting as described above.

Quantitative RT-PCR. Total RNA was extracted from macrophages using the RNeasy kit (Qiagen). cDNA was synthesized from $4 \mu \mathrm{g}$ total RNA using oligo (dT) and Superscript II (Invitrogen). cDNA (1 $\mu$ l) was subjected to quantitative PCR using the QuantiTect assay kit and primers for Fas (Invitrogen) and acidic ribosomal phosphoprotein (Arbp) as an internal control (Qiagen). The reactions were run on a MX4000 multiplex quantitative PCR system (Stratagene); the thermal profile settings were as follows: 40 cycles of $95^{\circ} \mathrm{C}$ for 15 minutes, $94^{\circ} \mathrm{C}$ for 15 seconds, $55^{\circ} \mathrm{C}$ for 30 seconds, and $72^{\circ} \mathrm{C}$ for 30 seconds; followed by disassociation at $95^{\circ} \mathrm{C}$ for 30 seconds and then $60^{\circ} \mathrm{C}$ for 1 minute. Relative fold changes of Fas or CaMKII $\gamma$ over Arbp control were determined using the $2^{-\Delta \Delta C \mathrm{CT}} \operatorname{method}(79)$. The following primers were used for PCR amplification of CaMKII isoforms according to Ang et al. (80): CaMKII $\alpha$ forward, 5'-TACAGTGAAGCTGATGCCAG-3'; CaMKII $\alpha$ reverse, $5^{\prime}$-TCGCACATCTTCGTGTAGGA-3'; CaMKII $\beta$ forward, 5'-TACAGTGAAGCTGATGCCAG-3'; CaMKII $\beta$ reverse, $5^{\prime}$-ACTCTTGGCTTGTTCCACCA-3'; CaMKII $\gamma$ forward, 5'-TACAGTGAAGCTGATGCCAG-3'; CaMKII $\gamma$ reverse, 5'-TTGACACCGCCATCTGACTT-3'; CaMKIIס forward, 5'-TACAGTGAAGCTGATGCCAG-3'; CaMKIIס reverse, 5'-GAGCACAGGCAGACAAACAT- $3^{\prime}$.

Measurement of cell surface proteins. The Cell Surface Labeling Kit (Pierce) was used to isolate cell surface proteins according to the manufacturer's instructions. The protocol was proportionately scaled down to be used with $60-\mathrm{mm}$ dishes. At the end of the incubation, macrophages were washed 2 times with ice-cold PBS followed by the addition of the biotin conjugate. The cells were incubated at $4{ }^{\circ} \mathrm{C}$ for 30 minutes on a rocking platform. The reaction was stopped by incubation with the kit's quenching solution for 1 minute. The cells were then scraped and centrifuged at $500 \mathrm{~g}$ for $3 \mathrm{~min}$ utes. The pellet was washed and lysed by sonication on ice with 5 burst of 1 second each, followed by an additional 30 -minute incubation on ice. The cell lysates were centrifuged at $10,000 \mathrm{~g}$ for 2 minutes, and the supernates were divided equally into 2 tubes. In one tube per condition, the lysates were incubated with NeurtAvidin gel with end-over-end rotation for 60 minutes at $4^{\circ} \mathrm{C}$. The slurry was washed 3 times, followed by incubation for 1 hour with water containing $50 \mathrm{mM}$ DTT and then centrifugation to isolate the eluted proteins. The material in the other tube per condition was used for whole-cell lysate analysis. Protein concentration was determined, and the material was subjected to SDS-PAGE and immunoblot analysis.

Measurement of CaMKII activity. CaMKII activity was assayed using a CaMKII assay kit from Millipore Corp. according to the manufacturer's instructions. After the cells were treated as indicated in the figure legends, they were lysed by a 5 -minute exposure to $1 \%$ Triton- $X$ in $50 \mathrm{mM}$ HEPES, $150 \mathrm{mM} \mathrm{NaCl}, 10$ mM Na pyrophosphate, $10 \mathrm{mM}$ EDTA, $10 \mathrm{mM}$ EGTA, $1 \mathrm{mM} \mathrm{Na}_{3} \mathrm{VO}_{4}, 50$ $\mathrm{mM} \mathrm{NaF}, 1 \mathrm{mM}$ PMSF, and $5 \mu \mathrm{g} / \mathrm{ml}$ leupeptin. Next, [ $\left.\gamma^{-32} \mathrm{P}\right] \mathrm{ATP}$ and the spe- cific CaMKII substrate peptide KKALRRQETVDAL were added to the lysate, and, after incubation for 10 minutes at $30^{\circ} \mathrm{C}$, the [ $\left.{ }^{32} \mathrm{P}\right]$-phosphorylated substrate was separated from the residual [ $\left.{ }^{32} \mathrm{P}\right] \mathrm{ATP}$ using P81 phosphocellulose paper and quantitated using a scintillation counter.

siRNA silencing of CaMKII $\gamma$. siRNA sequences against CaMKII $\gamma$ were generated by Qiagen. The target sequences were AACGTGGTACATAATGCTACA, CACAGTCACTCCTGAAGCTAA, and ATCATTAAGATCACAGAACAA for siRNAs 1,2 , and 3 , respectively. The siRNA was transfected into macrophages grown to 50\% confluency using Lipofectamine (Invitrogen) according to the manufacturer's instructions. A final mass of $50 \mathrm{pg}$ siRNA was added to each well. After 4 hours of transfection, the media were replaced, and 72 hours later, the indicated experiments were conducted.

Mitochondrial calcium uptake. For the FCCP-Fura-2 method, macrophages were plated on $25-\mathrm{mm}$ coverslips and grown to confluency for 4 days. On the day of the experiment, the macrophages were cholesterol loaded for 1 hour at $37^{\circ} \mathrm{C}$. The cells were then washed twice with HBSS wash solution (Hanks buffered saline, $250 \mu \mathrm{M}$ sulfinpyrazone, and $10 \mu \mathrm{g} / \mathrm{ml} 58035$ ) and stained with $5 \mu \mathrm{M}$ Fura-2 plus $0.08 \%$ Pluronic F-127 (Invitrogen) in wash solution. The cells were wrapped in foil and incubated at room temperature for 30 minutes. The Fura-2 solution was removed, and the cells were washed twice and incubated an additional 30 minutes in HBSS wash solution. Imaging was achieved using an inverted Nikon diaphot microscope equipped with a $\times 40$ objective. Sulfinpyrazone $(250 \mu \mathrm{M})$ was included in all solutions to prevent excretion of the Fura- 2 by macrophages. Images were taken every second for up to 1,600 seconds. During the imaging, $1 \mu \mathrm{M} \mathrm{FCCP}$ and then $10 \mu \mathrm{M}$ ionomycin were added at the indicated times. Fluorescence images (510-nm emission after alternate 340- and 380-nm excitation) were collected through a charge-coupled device camera (Photon Technology International), and the 340/380 ratio of 40-50 cells in these images was calculated.

For the Rhod-2 method, macrophages in phenol red-free medium were incubated on ice for 1 hour on a rocking platform with $10 \mu \mathrm{M}$ Rhod-2-AM (Invitrogen) 4 hours prior to the end of the experiment. The cells were then washed once with PBS, followed by incubation with the indicated experimental treatment media for 3 hours at $37^{\circ} \mathrm{C}$. Cells were imaged using a scanning confocal microscope (model LSM 510 META, $\times 40 / 1.3$ numerical aperture oil immersion lens; Carl Zeiss). The optical sections were estimated to be $1 \mu \mathrm{M}$ in thickness. The cells were excited using a 543-nm helium neon laser, and emitted fluoresce was detected through a 530-nm-long path filter. Quantification of the rhodamine signal was conducted using NIH Image J version $1.37 \mathrm{v}$ (http://rsb.info.nih.gov/ij/). Intensity values represent mean integrated fluorescence signal per cell.

Mitochondrial membrane potential. At the end of the indicated treatment periods, macrophages on glass-bottomed 35-mm culture dishes (Mat Tek) were washed twice with PBS and then stained with $100 \mu \mathrm{M}$ MitoTracker CMXRos (Invitrogen) for $15-30$ minutes at $37^{\circ} \mathrm{C}$. Fluorescent images were captured and quantified as detailed above for the Rhod- 2 method.

Isolation of mitochondria. Mitochondria were isolated using a QProteome Mitochondria Isolation Kit (Qiagen). Macrophages in 35-mm culture dishes were washed, lysed, and centrifuged at $1,000 \mathrm{~g}$ for 10 minutes at $4{ }^{\circ} \mathrm{C}$. The supernates were collected (cytosolic fraction), and the pellets were resuspended and sheared via passage 10 times through a 26 -gauge needle. These lysates were then centrifuged at $1,000 \mathrm{~g}$ for 10 minutes at $4^{\circ} \mathrm{C}$, and the supernatant fractions, containing the mitochondria, were centrifuged again at $6,000 \mathrm{~g}$ for 10 minutes at $4^{\circ} \mathrm{C}$. The pellets were resuspended in $1 \%$ SDS. The cytosolic fractions (above) were concentrated using acetone precipitation on ice for 1 hour, followed by centrifugation at $10,000 \mathrm{~g}$ for 10 minutes at $4{ }^{\circ} \mathrm{C}$ and resuspension of the pellets in $1 \%$ SDS. After assay of protein concentration, the fractions were subjected to SDS-PAGE and immunoblot analysis. 
Mouse model of ER stress. Camk2 $\mathrm{g}^{-1}$ and WT mice were injected i.v. in the tail vein with $2 \mathrm{mg} / \mathrm{kg}$ tunicamycin in sterile isotonic dextrose or with the same volume of vehicle control. Peritoneal macrophages were harvested 24 hours later, resuspended in DMEM plus 10\% FBS and 20\% LCM, incubated in tissue culture dishes for 30 minutes, and then assayed for apoptosis or mitochondrial membrane potential. At 3 days prior to tunicamycin treatment, all mice were injected i.p. with concanavalin $\mathrm{A}$ as described above. For the spleen and kidney studies, Camk2 $g^{/-}$and WT mice were injected with $1 \mathrm{mg} / \mathrm{kg}$ tunicamycin i.p. in sterile isotonic dextrose or with the same volume of vehicle control. The organs were harvested 48 hours after the tunicamycin injection, and cryosections were prepared. For the spleen study, TUNEL staining was performed using the In Situ Cell Death Detection Kit, TMR red (Roche Applied Science), followed by immunofluorescence staining using rat anti-CD68 (Serotec) followed by FITC-labeled goat antirat IgG (Santa Cruz Biotechnology Inc.). For the kidney study, TUNEL was conducted as above, followed by nuclear counterstaining with DAPI. Sections were mounted with ProLong Gold antifade reagent (Invitrogen), and images were captured using an Olympus IX-70 inverted microscope.

Renal function tests. Serum was collected after centrifuging clotted blood at $3,000 \mathrm{~g}$ for 20 minutes. The creatinine concentration in serum was measured using a commercially available kit (Bio-quant Inc.). The albumin concentration in the urine was measured by a competitive ELISA (Exocell) and normalized to urine creatinine levels, measured as described above.

Statistics. Results are expressed as mean $\pm \mathrm{SEM}, n=3$. Absent error bars signify SEM values smaller than the graphic symbols. ANOVA followed by Tukey post-test (GraphPad Prism 4 version 4.03) was used to determine statistical significance among all groups. A $P$ value less than 0.05 was considered significant.

1. Welihinda, A.A., Tirasophon, W., and Kaufman, R.J. 1999. The cellular response to protein misfolding in the endoplasmic reticulum. Gene Expr. 7:293-300.

2. Ron, D. 2002. Translational control in the endoplasmic reticulum stress response. J. Clin. Invest. 110:1383-1388.

3. Feng, B., et al. 2003. The endoplasmic reticulum is the site of cholesterol-induced cytotoxicity in macrophages. Nat. Cell Biol. 5:781-792.

4. Marciniak, S.J., et al. 2004. CHOP induces death by promoting protein synthesis and oxidation in the stressed endoplasmic reticulum. Genes Dev. 18:3066-3077.

5. Szegezdi, E., Logue, S.E., Gorman, A.M., and Samali, A. 2006. Mediators of endoplasmic reticulum stress-induced apoptosis. EMBO Rep. 7:880-885.

6. Wu, J., and Kaufman, R.J. 2006. From acute ER stress to physiological roles of the Unfolded Protein Response. Cell Death. Differ. 13:374-384.

7. Sekine, Y., Takeda, K., and Ichijo, H. 2006. The ASK1-MAP kinase signaling in ER stress and neurodegenerative diseases. Curr. Mol. Med. 6:87-97.

8. Oyadomari, S., Araki, E., and Mori, M. 2002. Endoplasmic reticulum stress-mediated apoptosis in pancreatic beta-cells. Apoptosis. 7:335-345.

9. Tabas, I. 2005. Consequences and therapeutic implications of macrophage apoptosis in atherosclerosis: the importance of lesion stage and phagocytic efficiency. Arterioscler. Thromb. Vasc. Biol 25:2255-2264.

10. Yao, P.M., and Tabas, I. 2000. Free cholesterol loading of macrophages induces apoptosis involving the fas pathway. J. Biol. Chem. 275:23807-23813.

11. Yao, P.M., and Tabas, I. 2001. Free cholesterol loading of macrophages is associated with widespread mitochondrial dysfunction and activation of the mitochondrial apoptosis pathway. J. Biol. Chem. 276:42468-42476.

12. Seimon, T., and Tabas, I. 2009. Mechanisms and consequences of macrophage apoptosis in atherosclerosis. J. Lipid Res. 50(Suppl.):S382-S387.

13. Feng, B., et al. 2003. Niemann-Pick C heterozygos-

\section{Acknowledgments}

We acknowledge Andrew R. Marks and his colleagues for assistance with the Fura-2 measurements; Connie Woo for assistance with the spleen and kidney experiments; and Lloyd Greene for helpful discussions. We thank Richard Flavell, David Levy, Richard M. Schultz, and Paula Stein for providing mice for this study. We thank Naoki Terasaka, in the laboratory of Alan Tall, for providing the human aortic endothelial cells. This work was supported by an NIH T32 postdoctoral training grant HL007343 to J.M. Timmins; American Heart Association Scientist Development Grant 0735594T to T.A. Seimon; US Army Medical Research and Materiel Command grant W81XWH-06-1-0212 to I. Tabas; NIH grants HL087123 and HL075662 to I. Tabas; Parkinson's Disease Foundation and NIH Udall Center Grant P50 NS038370 to C. Malagelada; Emmy Noether-DFG grant 2258/2-1 to J. Backs; and NIH grants HL079031, HL62494, and HL70250 and a grant from the Fondation Leducq to M.E. Anderson. E.N. Olson was supported by grants from the NIH, the Donald W. Reynolds Center for Clinical Cardiovascular Research, the Robert A. Welch Foundation, and the American Heart Association.

Received for publication May 27, 2009, and accepted in revised form July 1, 2009.

Address correspondence to: Ira Tabas, Department of Medicine, Columbia University, 630 West 168th Street, New York, New York 10032, USA. Phone: (212) 305-9430; Fax: (212) 305-4834; E-mail: iat1@columbia.edu. ity confers resistance to lesional necrosis and macrophage apoptosis in murine atherosclerosis. Proc. Natl. Acad. Sci. U. S. A. 100:10423-10428.

14. Li, Y., et al. 2004. Enrichment of endoplasmic reticulum with cholesterol inhibits SERCA2b activity in parallel with increased order of membrane lipids. Implications for depletion of ER calcium stores and apoptosis in cholesterol-loaded macrophages. J. Biol. Chem. 279:37030-37039.

15. DeVries-Seimon, T., et al. 2005. Cholesterol-induced macrophage apoptosis requires ER stress pathways and engagement of the type A scavenger receptor. J. Cell Biol. 171:61-73.

16. Zhou, J., Lhotak, S., Hilditch, B.A., and Austin, R.C. 2005. Activation of the unfolded protein response occurs at all stages of atherosclerotic lesion development in apolipoprotein E-deficient mice. Circulation. 111:1814-1821.

17. Gargalovic, P.S., et al. 2006. The unfolded protein response is an important regulator of inflammatory genes in endothelial cells. Arterioscler. Thromb. Vasc. Biol. 26:2490-2496.

18. Myoishi, M., et al. 2007. Increased endoplasmic reticulum stress in atherosclerotic plaques associated with acute coronary syndrome. Circulation. 116:1226-1233.

19. Han, S., et al. 2006. Macrophage insulin receptor deficiency increases ER stress-induced apoptosis and necrotic core formation in advanced atherosclerotic lesions. Cell Metab. 3:257-266.

20. Thorp, E., et al. 2009. Reduced apoptosis and plaque necrosis in advanced atherosclerotic lesions of Apoe-/- and Ldlr-/- mice lacking CHOP. Cell Metab. 9:474-481.

21. Boyce, M., and Yuan, J. 2006. Cellular response to endoplasmic reticulum stress: a matter of life or death. Cell Death Differ. 13:363-373.

22. Nakagawa, T., et al. 2000. Caspase-12 mediates endoplasmic-reticulum-specific apoptosis and cytotoxicity by amyloid- $\beta$. Nature. 403:98-103.

23. Seimon, T.A., Obstfeld, A., Moore, K.J., Golenbock,
D.T., and Tabas, I. 2006. Combinatorial pattern recognition receptor signaling alters the balance of life and death in macrophages. Proc. Natl. Acad. Sci. U. S. A. 103:19794-19799.

24. Manning-Tobin, J.J., et al. 2009. Loss of SR-A and CD36 activity reduces atherosclerotic lesion complexity without abrogating foam cell formation in hyperlipidemic mice. Arterioscler. Thromb. Vasc. Biol. 29:19-26

25. Lim, W.S., et al. 2008. STAT1 is critical for apoptosis in macrophages subjected to endoplasmic reticulum stress in vitro and in advanced atherosclerotic lesions in vivo. Circulation. 117:940-951.

26. Li, G., et al. 2009. Role or ERO1a-mediated stimulation of inositol 1,4,5-triphosphate receptor activity in endoplasmic reticulum stress-induced apoptosis. J. Cell Biol. In press.

27. Nagata, S. 1997. Apoptosis by death factor. Cell. 88:355-365.

28. Ortiz-Arduan, A., et al. 1996. Regulation of Fas and Fas ligand expression in cultured murine renal cells and in the kidney during endotoxemia. Am. J. Physiol. 271:F1193-F1201.

29. Miyano, O., Kameshita, I., and Fujisawa, H. 1992. Purification and characterization of a brain-specific multifunctional calmodulin-dependent protein kinase from rat cerebellum. J. Biol. Chem. 267:1198-1203.

30. Ishida, A., et al. 1998. Critical amino acid residues of AIP, a highly specific inhibitory peptide of calmodulin-dependent protein kinase II. FEBS Lett. 427:115-118.

31. Colbran, R.J. 1992. Regulation and role of brain calcium/calmodulin-dependent protein kinase II. Neurochem. Int. 21:469-497.

32. Erickson, J.R., et al. 2008. A dynamic pathway for calcium-independent activation of CaMKII by methionine oxidation. Cell. 133:462-474.

33. Ryu, E.J., et al. 2002. Endoplasmic reticulum stress and the unfolded protein response in cellular models of Parkinson's disease. J. Neurosci. 22:10690-10698. 
34. Cuschieri, J., Bulger, E., Garcia, I., Jelacic, S., and Maier, R.V. 2005. Calcium/calmodulin-dependent kinase II is required for platelet-activating factor priming. Shock. 23:99-106.

35. Storling, J., et al. 2005. Calcium has a permissive role in interleukin-1beta-induced c-jun $\mathrm{N}$-terminal kinase activation in insulin-secreting cells. Endocrinology. 146:3026-3036.

36. Lasham, A., Lindridge, E., Rudert, F., Onrust, R., and Watson, J. 2000. Regulation of the human fas promoter by YB-1, Puralpha and AP-1 transcription factors. Gene. 252:1-13.

37. Jia, L., et al. 2008. Critical roles for JNK, c-Jun, and Fas/FasL-Signaling in vitamin $\mathrm{E}$ analog-induced apoptosis in human prostate cancer cells. Prostate. 68:427-441.

38. Galluzzi, L., et al. 2007. Methods for the assessment of mitochondrial membrane permeabilization in apoptosis. Apoptosis. 12:803-813.

39. Lee, H.J., et al. 2007. The role of STAT1/IRF-1 on synergistic ROS production and loss of mitochondrial transmembrane potential during hepatic cell death induced by LPS/d-GalN. J. Mol. Biol. 369:967-984.

40. Rizzuto, R., et al. 2003. Calcium and apoptosis: facts and hypotheses. Oncogene. 22:8619-8627.

41. Deniaud, A., et al. 2008. Endoplasmic reticulum stress induces calcium-dependent permeability transition, mitochondrial outer membrane permeabilization and apoptosis. Oncogene. 27:285-299.

42. Giacomello, M., Drago, I., Pizzo, P., and Pozzan, T. 2007. Mitochondrial Ca2+ as a key regulator of cell life and death. Cell Death Differ. 14:1267-1274.

43. Thayer, S.A., and Miller, R.J. 1990. Regulation of the intracellular free calcium concentration in single rat dorsal root ganglion neurones in vitro. J. Physiol. 425:85-115.

44. Trollinger, D.R., Cascio, W.E., and Lemasters, J.J. 1997. Selective loading of Rhod 2 into mitochondria shows mitochondrial $\mathrm{Ca} 2+$ transients during the contractile cycle in adult rabbit cardiac myocytes. Biochem. Biophys. Res. Commun. 236:738-742.

45. Kirichok, Y., Krapivinsky, G., and Clapham, D.E. 2004. The mitochondrial calcium uniporter is a highly selective ion channel. Nature. 427:360-364.

46. Strack, S., Choi, S., Lovinger, D.M., and Colbran, R.J. 1997. Translocation of autophosphorylated calcium/ calmodulin-dependent protein kinase II to the postsynaptic density. J. Biol. Chem. 272:13467-13470.

47. Zinszner, H., et al. 1998. CHOP is implicated in programmed cell death in response to impaired function of the endoplasmic reticulum. Genes Dev. 12:982-995.

48. Kitamura, M. 2008. Endoplasmic reticulum stress in the kidney. Clin. Exp. Nephrol. 12:317-325.

49. Tajiri, S., et al. 2006. CHOP is involved in neuronal apoptosis induced by neurotrophic factor deprivation. FEBS Lett. 580:3462-3468.

50. Oyadomari, S., et al. 2002. Targeted disruption of the Chop gene delays endoplasmic reticulum stressmediated diabetes. J. Clin. Invest. 109:525-532.

51. Song, B., Scheuner, D., Ron, D., Pennathur, S., and Kaufman, R.J. 2008. Chop deletion reduces oxidative stress, improves beta cell function, and promotes cell survival in multiple mouse models of diabetes. J. Clin. Invest. 118:3378-3389.

52. Kim, I., Xu, W., and Reed, J.C. 2008. Cell death and endoplasmic reticulum stress: disease relevance and therapeutic opportunities. Nat. Rev. Drug Discov. 7:1013-1030.

53. Ishida, A., Sueyoshi, N., Shigeri, Y., and Kameshita, I. 2008. Negative regulation of multifunctional $\mathrm{Ca} 2+$ / calmodulin-dependent protein kinases: physiological and pharmacological significance of protein phosphatases. Br. J. Pharmacol. 154:729-740.

54. Kim, H.S., and Lee, M.S. 2007. STAT1 as a key modulator of cell death. Cell Signal. 19:454-465.

55. Vila-Petroff, M., et al. 2007. CaMKII inhibition protects against necrosis and apoptosis in irreversible ischemia-reperfusion injury. Cardiovasc. Res. 73:689-698.

56. Mao, W., et al. 2007. Cardiomyocyte apoptosis in autoimmune cardiomyopathy: mediated via endoplasmic reticulum stress and exaggerated by norepinephrine. Am. J. Physiol. Heart Circ. Physiol. 293:H1636-H1645.

57. Zhu, W., et al. 2007. Activation of CaMKIIdeltaC is a common intermediate of diverse death stimuliinduced heart muscle cell apoptosis. J. Biol. Chem. 282:10833-10839.

58. Fladmark, K.E., et al. 2002. Ca2+/calmodulin-dependent protein kinase II is required for microcystininduced apoptosis. J. Biol. Chem. 277:2804-2811.

59. Laabich, A., Li, G., and Cooper, N.G. 2001. Characterization of apoptosis-genes associated with NMDA mediated cell death in the adult rat retina. Brain Res. Mol. Brain Res. 91:34-42.

60. Seimon, T.A., et al. 2009. Macrophage deficiency of p38alpha MAPK promotes apoptosis and plaque necrosis in advanced atherosclerotic lesions in mice. J. Clin. Invest. 119:886-898.

61. Kaplin, A.I., Ferris, C.D., Voglmaier, S.M., and Snyder, S.H. 1994. Purified reconstituted inositol 1, 4, 5-trisphosphate receptors. Thiol reagents act directly on receptor protein. J. Biol. Chem. 269:28972-28978.

62. Biswas, G., et al. 1999. Retrograde $\mathrm{Ca} 2+$ signaling in $\mathrm{C} 2 \mathrm{C} 12$ skeletal myocytes in response to mitochondrial genetic and metabolic stress: a novel mode of inter-organelle crosstalk. EMBO J. 18:522-533.

63. Pizzo, P., and Pozzan, T. 2007. Mitochondria-endoplasmic reticulum choreography: structure and signaling dynamics. Trends Cell Biol. 17:511-517.

64. Grueter, C.E., et al. 2006. L-type Ca2+ channel facilitation mediated by phosphorylation of the beta subunit by CaMKII. Mol. Cell. 23:641-650.

65. Lee, T.S., et al. 2006. Calmodulin kinase II is involved in voltage-dependent facilitation of the L-type Cav1.2 calcium channel: Identification of the phosphorylation sites. J. Biol. Chem. 281:25560-25567.

66. Wehrens, X.H., Lehnart, S.E., Reiken, S.R., and Marks, A.R. 2004. Ca2+/calmodulin-dependent protein kinase II phosphorylation regulates the cardiac ryanodine receptor. Circ. Res. 94:e61-e70.

67. Rodriguez, P., Bhogal, M.S., and Colyer, J. 2003.
Stoichiometric phosphorylation of cardiac ryanodine receptor on serine 2809 by calmodulin-dependent kinase II and protein kinase A. J. Biol. Chem. 278:38593-38600.

68. Backs, J., et al. 2009. The delta isoform of CaM kinase II is required for pathological cardiac hypertrophy and remodeling after pressure overload. Proc. Natl. Acad. Sci. U. S. A. 106:2342-2347.

69. Li, Y., et al. 2005. Free cholesterol-loaded macrophages are an abundant source of tumor necrosis factoralpha and interleukin-6: model of NF-kappaB- and map kinase-dependent inflammation in advanced atherosclerosis. J. Biol. Chem. 280:21763-21772.

70. Zhang, R., et al. 2005. Calmodulin kinase II inhibition protects against structural heart disease. Nat. Med. 11:409-417.

71. Khoo, M.S., et al. 2006. Death, cardiac dysfunction, and arrhythmias are increased by calmodulin kinase II in calcineurin cardiomyopathy. Circulation. 114:1352-1359.

72. Choy, J.C., Granville, D.J., Hunt, D.W., and McManus, B.M. 2001. Endothelial cell apoptosis: biochemical characteristics and potential implications for atherosclerosis. J. Mol. Cell Cardiol. 33:1673-1690.

73. Havel, R.J., Eder, H., and Bragdon, J. 1955. The distribution and chemical composition of ultracentrifugally reported lipoproteins in human serum. J. Clin. Invest. 34:1345-1353.

74. Basu, S.K., Goldstein, J.L., Anderson, R.G.W., and Brown, M.S. 1976. Degradation of cationized low density lipoprotein and regulation of cholesterol metabolism in homozygous familial hypercholesterolemia fibroblasts. Proc. Natl. Acad. Sci. U. S. A. 73:3178-3182.

75. Yang, D.D., et al. 1998. Differentiation of CD4+ T cells to Th1 cells requires MAP kinase JNK2. Immunity. 9:575-585.

76. Durbin, J.E., Hackenmiller, R., Simon, M.C., and Levy, D.E. 1996. Targeted disruption of the mouse Stat 1 gene results in compromised innate immunity to viral disease. Cell. 84:443-450.

77. Sakai, K., and Miyazaki,J. 1997. A transgenic mouse line that retains Cre recombinase activity in mature oocytes irrespective of the cre transgene transmission. Biochem. Biophys. Res. Commun. 237:318-324.

78. Malagelada, C., Ryu, E.J., Biswas, S.C., Jackson-Lewis, V., and Greene, L.A. 2006. RTP801 is elevated in Parkinson brain substantia nigral neurons and mediates death in cellular models of Parkinson's disease by a mechanism involving mammalian target of rapamycin inactivation. J. Neurosci. 26:9996-10005.

79. Livak, K.J., and Schmittgen, T.D. 2001. Analysis of relative gene expression data using real-time quantitative PCR and the 2(-Delta Delta C(T)) Method. Methods. 25:402-408.

80. Ang, E.S., et al. 2007. Calcium/calmodulin-dependent kinase activity is required for efficient induction of osteoclast differentiation and bone resorption by receptor activator of nuclear factor kappa B ligand (RANKL). J. Cell. Physiol. 212:787-795. 